\title{
SOME REMARKS ON LAGRANGIAN AND HAMILTONIAN FORMALISMS, RELATED TO INFINITE-DIMENSIONAL DYNAMICAL SYSTEMS WITH SYMMETRIES
}

\author{
A.PrykaRPatsky, R.SAMULIAK \\ Institule for Applied Problems of Mechanics and Mathematics \\ Ukrainian National Academy of Sciences, \\ 36 Naukova St., 290060 Lviv-60, Ukraine \\ D.BLACKMORE \\ New Jersey Institute of Technology \\ University Heights, Newark, NJ 07102-1982, USA \\ W.StRAMPP, YU.SYDORENKO \\ GH-Universität Kassel, FB-17 Matematik, \\ Hollandische Str. 36, BRD
}

Received June 29, 1994

\begin{abstract}
A description of Lagrangian and Hamiltonian formalisms strictly obtained from the invariance structure of given monlinear dynamical systems on the infinite-dimensional functional manifold is presented. The basic ideas used, in order to formulate the canonical symplectic structure, are borrowed from the Cartan's theory of differential systems on associated jet-manifolds. The symmetry structure reduced on the invariant submanifolds of critical points of some nonlocal EulerLagrange functional is described thoroughly for both differential and differential discrete dynamical systems.
\end{abstract}

\section{Introduction}

A fundamental problem in modern theory of infinite-dimensional dynamical systems is that of an invariant reduction them upon some invariant submanifolds with enough rich mathematical structures to treat their properties analytically. The first approaches to these problems were suggested still at the late times of the preceding century, in the classical papers by S.Lie, J.Liouville, J.Lagrange, V.R.Hamilton, J.Poisson and E.Cartan. They introduced for the first time the important concepts of symmetry, conservation law, symplectic, Poisson and Hamiltonian structures as well as invariant reduction procedure, which appeared to be extremely useful for proceeding studies. These notions were widely generalized further by Souriau [21], Marsden and Weinstein [34,20], Lax [3], Bogoyavlensky and Novikov [7], and by a lot of other researchers $[8,10-13]$. It seems worthwhile to mention here also the rather recent studies in $[22-29]$, where the special reduction methods were built for the integrable nonlinear dynamical systems on both 
functional and operational manifolds. In the present paper we describe in detail the reduction procedure for infinite dimensional dynamical systems upon the invariant set of critical points of some global invariant functional. The method uses the Cartan's differential-geometric treating of differential ideas in Grassmann algebra over the associated jet-manifold. As one of main results, we show also that both the reduced dynamical systems and their symmetries, generate the Hamiltonian flows on the invariant critical submanifolds of local and nonlocal functionals with respect to the canonical symplectic structure upon it. These results are generalized for the case of differential-difference dynamical systems being given on discrete infinitedimensional manifolds. The direct procedure to construct the invariant Lagrangian functionals for a given apriori Lax-type integrable dynamical system is presented for both the differential and the differential-difference cases of the manifold $M$. Some remarks on the Lagrangian and Hamiltonian formalisms concerning infinite-dimensional dynamical systems with symmetries are given.

\section{General setting}

We are interested in treating a given nonlinear dynamical system

$$
d u / d t=K[u]
$$

with respect to an evolution parameter $t \in \mathbb{R}$ on the infinite-dimensional functional manifold $M \subset C^{(\infty)}\left(\mathbb{R} ; \mathbb{R}^{m}\right)$, possessing two additional ingredients: a homogenous conservation law $\mathcal{L} \in D(M)$ and a number of homogenous symmetries $d u / d t_{j}=K_{j}[u], \quad j=\overline{1, k}$, with evolution parameters $t_{j} \in \mathbb{R}$. The dynamical system (2.1) is notsupposed to be Hamiltonian, all the maps $K, K_{j}: M \rightarrow T(M), j=\overline{1, k}$ being considered smooth and well-defined on $M$.

To pose the problem to be discussed further more definitely, let us involve the jet-manifold $J^{(\infty)}\left(\mathbb{R} ; \mathbb{R}^{m}\right)$ that is locally isomorphic to the functional manifold $M$ mentioned above. This means the following: the vector field (2.1) on $M$ is completely equivalent to that on the jet-manifold $J^{(\infty)}\left(\mathbb{R} ; \mathbb{R}^{m}\right)$ via the representation $[1,2]$

$$
\begin{aligned}
(M & \ni u \rightarrow K[u]) \stackrel{j e t}{\longrightarrow}\left(K\left(u, u^{(1)}, \ldots, u^{(n+1)}\right) \leftarrow\right. \\
& \left.\leftarrow\left(x ; u, u^{(1)}, \ldots, u^{(\infty)}\right) \in J^{(\infty)}\left(\mathbb{R} ; \mathbb{R}^{m}\right)\right),
\end{aligned}
$$

where $n \in \mathbb{Z}_{+}$- fixed, $x \in \mathbb{R}$ - the function parameter of the jet-bundle.

$J^{(\infty)}\left(\mathbb{R} ; \mathbb{R}^{m}\right) \stackrel{\pi}{\longrightarrow} \mathbb{R}, \pi$ - the projection on the base $\mathbb{R}$. Let us allow also that the smooth functional $\mathcal{L} \in D(M)$ is a conservation law of the dynamical system (2.1), that is $d \mathcal{L} / d t=0$ along orbits of (2.1) for all $t \in \mathbb{R}$. Due to the jet-representation (2.2) we can write the density of the functional $\mathcal{L} \in D(M)$ in the following form:

$$
\mathcal{L}=\int_{\mathbb{R}} d x \mathcal{L}[u]
$$

$$
(\mathbb{R} \ni \mathcal{L}[u]) \stackrel{\text { jet }}{\longrightarrow}\left(\mathcal{L}\left(u, u^{(1)}, \ldots, u^{(N+1)}\right) \in \mathbb{R}\right),
$$

where the number $N \in \mathbb{Z}_{+}$is fixed. Besides, we are going to assume the functional (2.3) as non-degenerated in the sense that Hessian of $\mathcal{L}$ : $J^{(N+1)}\left(\mathbb{R} ; \mathbb{R}^{m}\right) \rightarrow \mathbb{R}$ has nonvanishing determinant: det $\left\|\frac{\partial^{2} \mathcal{L}\left(u, u^{(1)}, \ldots, u^{(N+1)}\right)}{\partial u^{(N+1)} \partial u^{(N+1)}}\right\| \neq$ 0 . 


\section{Lagrangian reduction}

Consider now the set of critical points $M_{n} \subset M$ of the functional $\mathcal{L} \in D(M)$ :

$$
M_{N}=\{u \in M: \operatorname{grad} \mathcal{L}[u]=0\}
$$

where, due to $(2.2), \operatorname{grad} \mathcal{L}[u]:=\delta \mathcal{L}\left(u, \ldots, u^{(N+1)}\right) / \delta u$ - the Euler variational derivative. As proved by Lax [3], the manifold $M_{N} \subset M$ is smoothly imbedded well-defined one due to the condition $\operatorname{Hess\mathcal {L}} \neq 0$. Besides, the manifold $M_{N}$ is invariant in relation to the initial dynamical system (2.1). This means in particular that Lie-derivative of any field $X: M \rightarrow T(M)$, tangent to the manifold $M_{N}$, with respect to the vector field (2.1) is again tangent to $M_{N}$, that is, from

$$
X[u] \in T_{u}\left(M_{N}\right) \Rightarrow[K, X][u] \in T_{u}\left(M_{N}\right)
$$

for all $u \in M_{N}$. Here we are at the point to begin with a study the intrinsic structure of the manifold $M_{N} \subset M$ within the geometric Cartan's theory developed on the jet-manifold $J^{(\infty)}\left(\mathbb{R} ; \mathbb{R}^{m}\right)[2-5]$. Let us define an ideal $I(\xi) \subset$ $\Lambda\left(J^{(\infty)}\right)$, generated by the vector one-forms $\xi^{j}=d u^{(j)}-u^{(j+1)} d x, \quad j \in \mathbb{Z}_{+}$, which are canceled the vector field $d / d x$ on the jet-manifold $J^{(\infty)}\left(\mathbb{R} ; \mathbb{R}^{m}\right)$ :

$$
i_{\frac{d}{d \cdot x}} \xi^{j}=0, \quad j \in \mathbb{Z}_{+},
$$

where $x$ belongs to the jet-bundle base $\mathbb{R}, \quad i_{\frac{d}{d x}}$ - the intrinsic derivative,

$$
\frac{d}{d x}=\frac{\partial}{\partial x}+\sum_{j \in \mathbb{Z}_{+}}\left\langle u^{(j+1)}, \frac{\partial}{\partial u^{(j)}}\right\rangle,
$$

where $\langle.,$.$\rangle - the standard scalar product in \mathbb{R}^{m}$. The vector field $(2.1)$ on the jet-manifold $J^{(\infty)}(\mathbb{R} ; \mathbb{R})$ has the analogous representation:

$$
\frac{d}{d t}=\frac{\partial}{\partial t}+\sum_{j \in \mathbb{Z}_{+}}\left\langle K^{(j)}, \frac{\partial}{\partial u^{(j)}}\right\rangle
$$

where, by definition, $K^{(j)}:=\frac{d^{j}}{d x^{j}} K, j \in \mathbb{Z}_{+}$. The problem arises: how to build the intrinsic variables on the manifold $M_{N} \subset M$ from the jet-manifold coordinates on $\left.J^{(\infty)}\left(\mathbb{R} ; \mathbb{R}^{m}\right)\right)$ ? To proceed to the solution of the problem above, let us study the 1 -form $d \mathcal{L} \in \Lambda^{1}\left(J^{(\infty)}(\mathbb{R} ; \mathbb{R})\right)$ as that defined on the submanifold $M_{N} \subset M$. We have the following chain of identities in the Grassmann subalgebra $\Lambda\left(J^{(2 N+2)}\left(\mathbb{R} ; \mathbb{R}^{m}\right)\right)$ :

$$
\begin{aligned}
d \mathcal{L} & =d\left(i_{\frac{d}{d x}} \mathcal{L} d x\right)=d i_{\frac{d}{d x}}\left(\mathcal{L} d x+\sum_{j=0}^{N}\left\langle p_{j}, \xi^{j}\right\rangle\right) \\
& =\left(d i_{\frac{d}{d x}}+i_{\frac{d}{d x}} d\right) \cdot\left(\mathcal{L} d x+\sum_{j=0}^{N}\left\langle p_{j}, \xi^{j}\right\rangle\right)-i_{\frac{d}{d x}} d\left(\mathcal{L} d x+\sum_{j=0}^{N}\left\langle p_{j}, \xi^{j}\right\rangle\right)
\end{aligned}
$$

where $p_{j}: J^{(2 N+2)}\left(\mathbb{R} ; \mathbb{R}^{m}\right) \rightarrow \mathbb{R}^{m}, j=\overline{0, N}$,- - some still undefined vectorfunctions. Requiring now that 2 -form $d\left(\mathcal{L} d x+\sum_{j=0}^{N}\left\langle p_{j}, \xi^{j}\right\rangle\right)$ not to depend differentials $d u^{(j)}, j=\overline{1, N+1}$, with respect to the modulus $I(\xi) \subset$ 
$\Lambda\left(J^{(\infty)}\left(\mathbb{R} ; \mathbb{R}^{m}\right)\right)$ :

$$
i_{\frac{\partial}{\partial u}(j)}\left(d \mathcal{L} \wedge d x+\sum_{k=0}^{N}\left\langle d p_{k} \wedge \xi^{k}\right\rangle\right) \in I(\xi),
$$

we can determine the vector-functions $p_{j} \in \mathbb{R}^{m}, \quad j=\overline{0, N}$. As a result we obtain the following recurrent relations;

$$
\frac{d p_{j}}{d x}+p_{j-1}=\frac{\partial \mathcal{L}}{\partial u^{(j)}}
$$

for $j=\overline{1, N+1}$, setting $p_{-1}=0=p_{N+1}$ by definition. The unique solution to $(3.7)$ is made by the following expressions, $j=\overline{0, N}$ :

$$
p_{j}=\sum_{k=0}^{N}(-1)^{k} \frac{d^{k}}{d x^{k}} \frac{\partial \mathcal{L}}{\partial u^{(j+k+1)}} .
$$

Thereby we have got, owing (3.5) and (3.6), the final representation for the differential $d \mathcal{L}$ :

$$
\begin{aligned}
d \mathcal{L} & =\frac{d}{d x}\left(\left[\mathcal{L}-\sum_{j=0}^{N}\left\langle p_{j}, u^{(j+1)}\right\rangle\right] d x\right)= \\
& \left.=\frac{d}{d x}\left(\sum_{j=0}^{N}\left\langle p_{j}, d u^{(j)}\right\rangle\right]\right)+\langle\operatorname{grad} \mathcal{L}[u], d u\rangle,
\end{aligned}
$$

where $\frac{d}{d x}:=d i_{\frac{d}{d x}}+i_{\frac{d}{d x}} d-$ the Lie-derivative along the vector field $\frac{d}{d x}$, $\mathcal{L}[u]:=\delta \mathcal{L} / \delta u$, as it was mentioned above in the chapter 2 . Below we intend to treat the representation (3.9) on the question of a symplectic structure arisen from the above analysis on the invariant submanifold $M_{N} \subset M$.

\section{Symplectic analysis and Hamiltonian settings}

Let us put into the expression (3.9) the condition $\operatorname{grad} \mathcal{L}[u]=0$ for all $u \in M_{N}$. Then the following equality is satisfied:

$$
d \mathcal{L}=\frac{d}{d x} \alpha^{(1)}, \quad \alpha^{(1)}=\sum_{j=0}^{N}\left\langle p_{j}, d u^{(j)}\right\rangle,
$$

since the function $h^{(x)}:=\sum_{j=0}^{N}\left\langle p_{j}, \frac{d u^{(j)}}{d x}\right\rangle-\mathcal{L}\left(u, \ldots, u^{(N+1)}\right)$ satisfies the condition $d h / d x=0$ for all $x \in \mathbb{R}$, owing to the relations (3.7). Taking now the external derivative of (4.1), we obtain that

$$
\frac{d}{d x} \Omega^{(2)}=0, \quad \Omega^{(2)}=d \alpha^{(1)},
$$

where we used the well known identity $d \cdot \frac{d}{d x}=\frac{d}{d x} \cdot d$. From (4.2) we can conclude that vector field $d / d x$ on the submanifold $M_{N} \subset M$ is a Hamiltonian one in the respect of the canonical symplectic structure $\Omega^{(2)}=$ 
$\sum_{j=0}^{N}\left\langle d p_{j} \wedge d u^{(j)}\right\rangle$. It is a very simple exercise to state that the function $h^{(x)}: J^{(2 N+2)}\left(\mathbb{R} ; \mathbb{R}^{m}\right) \rightarrow \mathbb{R}$ defined above is playing a role as corresponding Hamiltonian one for the vector field $d / d x$ on $M_{N}$, i.e. the equation

$$
d h^{(x)}=-i_{\frac{d}{d x}} \Omega^{(2)}
$$

is true on $M_{N}$. Therefore, we have got the following theorem to be true.

Theorem 1 The critical submanifold $M_{N} \subset M$ defined by (3.1) for a given nondegenerate smooth functional $\mathcal{L} \in D(M) \subset D\left(J^{(N+1)}\left(\mathbb{R} ; \mathbb{R}^{m}\right)\right)$, being imbedded in the jet-manifold $J^{(\infty)}\left(\mathbb{R} ; \mathbb{R}^{m}\right)$, carries the canonical symplectic structure, with reference to which the induced vector field $d / d x$ on $M_{N}$ is the Hamiltonian one.

The theorem analogous to the above was stated before via different manners by many authors $[8,9]$. Our different derivation presented here is both much simpler and more constructive, giving rise to all ingredients of symplectic theory, being stemmed from the imbedding of the invariant submanifold $M_{N}$ into the jet-manifold.

Now we are going to proceed further to studying the vector field (2.1) on the manifold $M_{N} \subset M$ endowed with the symplectic structure $\Omega^{(2)} \in$ $\Lambda^{2}\left(J^{(N+1)}\left(\mathbb{R} ; \mathbb{R}^{m}\right)\right)$, the latter being built via the formula $(4.2)$.

We have the following implicating identities:

$$
\begin{aligned}
& \frac{d \mathcal{L}}{d t}=0 \Rightarrow \quad\langle\operatorname{grad} \mathcal{L}[u], K[u]\rangle=-\frac{d h^{(t)}}{d x} \\
& \frac{d \mathcal{L}}{d x}=0 \Rightarrow\left\langle\operatorname{grad} \mathcal{L}[u], \frac{d u}{d x}\right\rangle=-\frac{d h^{(x)}}{d x}
\end{aligned}
$$

where functions $h^{(t)}$ and $h^{(x)}$ serve as corresponding Hamiltonian ones for the vector fields $d / d t$ and $d / d x$. This means that the following equations are true:

$$
d h^{(x)}=-i_{\frac{d}{d x}} \Omega^{(2)}, \quad d h^{(t)}=-i_{\frac{d}{d t}} \Omega^{(2)} .
$$

To prove the above statement (4.5), we shall build the following quantities (for the vector field $d / d x$ at first):

$$
d \cdot i_{\frac{d}{d x}}\langle\operatorname{grad} \mathcal{L}[u], d u\rangle=-\frac{d}{d x}\left(d h^{(x)}\right)
$$

- from (4.4), and

$$
i_{\frac{d}{d x}} \cdot d\langle\operatorname{grad} \mathcal{L}[u], d u\rangle=-\frac{d}{d x}\left(i_{\frac{d}{d x}} \Omega^{(2)}\right)
$$

- from (3.9), where the identity $\left[i_{\frac{d}{d x}}, \frac{d}{d x}\right]=0$ is preliminary used. Adding now the expression (4.6) and (4.7) entails the following one:

$$
\frac{d}{d x}\langle\operatorname{grad} \mathcal{L}[u], d u\rangle=-\frac{d}{d x}\left(d h^{(x)}+i_{\frac{d}{d x}} \Omega^{(2)}\right)
$$

for all $x \in \mathbb{R}$ and $u \in M$. Since $\operatorname{grad} L[u]=0$ for all $u \in M_{N}$, we obtain from (4.8) that the first equality in (4.5) is valid in the case of the vector field $d / d x$ reduced on $M_{N}$. The analogous procedure fits also for the vector 
field $d / d t$ reduced on the manifold $M_{N} \subset M$. The even difference of the procedure above stems from the condition on vector fields $d / d t$ and $d / d x$ to be commutative, $[d / d t, d / d x]=0$ what engenders the needed identity $\left[i_{\frac{d}{d t}}, \frac{d}{d x}\right] \equiv i_{\left[\frac{d}{d t}, \frac{d}{d x}\right]}=0$ as a simple consequence of the considerable above. There upon we have stated the validity of equations (4.5) completely.

Theorem 2. Dynamical systems $d / d t$ and $d / d x$ reduced on the invariant submanifold $M_{N} \subset M(3.1)$ are Hamiltonian ones with the corresponding Hamiltonian functions built from the equations (4.4) in the unique way.

By the way we have stated also that the Hamiltonian functions $h^{(x)}$ and $h^{(t)}$ on the submanifold $M_{N} \subset M$ are commuting with each other, that is $\left\{h^{(t)}, h^{(x)}\right\}=0$. This indeed follows from the equalities (4.4), since $\left\{h^{(t)}, h^{(x)}\right\}=\frac{d h^{(x)}}{d t}=-\frac{d h^{(t)}}{d x} \equiv 0$ upon the manifold $M_{N} \subset M$.

\section{Symmetry invariance}

Let us consider now any vector field $K_{j}: M \rightarrow T(M), j \in \overline{1, k}$ being a symmetry field for the given vector field (2.1), i.e. $\left[K, K_{j}\right]=0, j \in \overline{1, k}$. As the conservation law $\mathcal{L} \in D(M)$ for the vector field (2.1) should not be relevant for the vector field $K_{j}, \quad j \in \overline{1, k}$, the manifold $M_{N} \subset M$ should not be invariant also with respect to that vector field. Therefore, if a vector field $X \in T\left(M_{N}\right)$, the vector field $\left[K_{j}, X\right] \notin T\left(M_{N}\right)$ in general, if $\frac{d}{d t_{3}}, \quad j \in \overline{1, k}$, is chosen as symmetry of (2.1). Let us consider the following identity for some there existing function $\tilde{h}_{j}: J^{(2 N+2)}\left(\mathbb{R} ; \mathbb{R}^{m}\right) \rightarrow \mathbb{R}, \quad j \in \overline{1, k}$, originated by the condition $\left[\frac{d}{d t}, \frac{d}{d t_{j}}\right]=0, \quad j \in \overline{1, k}$, on $M$ :

$$
\frac{d}{d t}{ }^{\frac{d}{d t_{j}}}\langle\operatorname{grad} \mathcal{L}[u], d u\rangle=-\frac{d \tilde{h}_{j}[u]}{d x} .
$$

Lemma 1 .The function $\tilde{h}_{j}[u], \quad j \in \overline{1, k}$, reduced on the invariant submanifold $M_{N} \subset M$ turns into constant. This constant can be chosen obviously as a zero one.

Proof. We have: $\left[\frac{d}{d t}, i_{\frac{d}{d t_{j}}}\right]=0, \quad j \in \overline{1, k}$, there upon

$$
\begin{gathered}
i_{{\frac{d}{d t_{j}}}^{\prime}}\left(i_{\frac{d}{d t}} d+d i_{\frac{d}{d t}}\right)\langle\operatorname{grad} \mathcal{L}[u], d u\rangle=-\frac{d \tilde{h}_{j}}{d x} \Rightarrow \\
\Rightarrow i_{\frac{d}{d t_{j}}}\left(i_{\frac{d}{d t}} d\langle\operatorname{grad} \mathcal{L}[u], d u\rangle+d i_{\frac{d}{d t}}\langle\operatorname{grad} \mathcal{L}[u], d u\rangle\right) \\
=-i_{\frac{d}{d t_{j}}} i_{\frac{d}{d t}} \frac{d}{d x} \Omega^{(2)}-i_{\frac{d}{d t_{j}}} \frac{d}{d x}\left(d h^{(+)}\right)=-\frac{d}{d x} i_{\frac{d}{d t_{j}}}\left(i_{\frac{d}{d t}} \Omega^{(2)}+d h^{(t)}\right)=-\frac{d \tilde{h}_{j}}{d x},
\end{gathered}
$$

whence we gain that upon whole jet-manifold $M \subset J^{\infty}\left(\mathbb{R} ; \mathbb{R}^{m}\right)$ the following identity holds:

$$
i_{\frac{d}{d t_{j}}}\left(d h^{(t)}+i_{\frac{d}{d t}} \Omega^{(2)}\right)=\tilde{h}_{j}: \bmod \mathbb{R} .
$$

Since on the submanifold $M_{N} \subset M i_{\frac{d}{d t}} \Omega^{(2)}=-d h^{(t)}$, we find that $\tilde{h}_{j} \equiv$ $0, j \in \overline{1, k}$, that proves the lemma. 
Note 1.The result above could be stated also using the standard operatorial-functional calculus of [8]. Indeed,

$$
\begin{aligned}
\frac{d}{d t} i_{\frac{d}{d t_{i}}} & \langle\operatorname{grad} \mathcal{L}[u], d u\rangle \\
& =\frac{d}{d t}\left\langle\operatorname{grad} \mathcal{L}[u], K_{j}[u]\right\rangle \\
& =\left\langle\frac{d}{d t} \operatorname{grad} \mathcal{L}[u], K_{j}[u]\right\rangle+\left\langle\operatorname{grad} \mathcal{L}[u], \frac{d}{d t} K_{j}[u]\right\rangle \\
& =\left\langle-K^{\prime *} \cdot \operatorname{grad} \mathcal{L}[u], K_{j}[u]\right\rangle+\left\langle\operatorname{grad} \mathcal{L}[u], K_{j}^{\prime} \cdot K[u]\right\rangle \\
& =-\left\langle K^{\prime *} \cdot \operatorname{grad} \mathcal{L}[u], K_{j}[u]\right\rangle+\left\langle\operatorname{grad} \mathcal{L}[u], K^{\prime} \cdot K_{j}[u]\right\rangle \\
& =-\frac{d}{d x} \mathcal{H}_{j}\left(\operatorname{grad} \mathcal{L}[u], K_{j}[u]\right)=-\frac{d \tilde{h}_{j}[u]}{d x}
\end{aligned}
$$

There the bilinear form $\mathcal{H}_{j}(\because), \quad j \in \overline{1, k}$, is found via the usual definition of the adjoined operator $K_{j}^{\prime *}$ for a given operator $K_{j}^{\prime}: L_{2} \rightarrow L_{2}$ with respect to the natural scalar bracket $(\cdot, \cdot)$

$$
\left(K_{j}^{\prime *} a, b\right):=\left(a, K_{j}^{\prime} b\right), \quad(a, b):=\int_{\mathbb{R}} d x\langle a, b\rangle,
$$

whence we simply derive:

$$
\left\langle K_{j}^{\prime *} a, b\right\rangle-\left\langle a, K_{j}^{\prime} b\right\rangle=d \mathcal{H}_{j}(a, b)
$$

for all $a, b \in L_{2}$. Therefore, we can establish identity $\tilde{h}_{j}[u]=\mathcal{H}_{j}(\operatorname{grad} \mathcal{L}[u]$, $\left.K_{j}[u]\right)$ for all $u \in M$. If $u \in M_{N} \subset M$, we therewith obtain that $\tilde{h}_{j}[u] \equiv$ $0, j \in \overline{1, k}$, what was needed to prove.

As a result of the Lemma proved above is the following: the function $\tilde{h}_{j}[u], \quad j \in \overline{1, k}$, can not serve as the nontrivial Hamiltonian one for the dynamical system $d / d t_{j}, \quad j \in \overline{1, k}$, on the submanifold $M_{N} \subset M$. To overcome this difficulty we assume the invariant submanifold $M_{N} \subset M$ to possess some additional symmetries $d / d t_{j}, j \in \overline{1, k}$, which satisfy the following characteristic criteria: $L_{\frac{d}{d t_{3}}} \operatorname{grad} \mathcal{L}[u]=0, \quad j \in \overline{1, k}$, for all $u \in M_{N}$. This means that for $j \in \overline{1, k}$

$$
L_{\frac{d}{d t_{3}}} \operatorname{grad} \mathcal{L}[u]=G_{j}(\operatorname{grad} \mathcal{L}[u]),
$$

where $G_{j}(\cdot), \quad j \in \overline{1, k}$ - some linear vector-functionals on $T^{*}(M)$. Otherwise, the equation (5.6) is equivalent to the following:

$$
i_{\mathrm{dt}_{i}}\langle\operatorname{grad} \mathcal{L}[u], d u\rangle=-\frac{d h_{j}[u]}{d x}+g_{j}(\operatorname{grad} \mathcal{L}[u]),
$$

where $g_{j}(\cdot), \quad j \in \overline{1, k}$ - some scalar linear functionals on $T^{*}(M)$. From (3.9) and (5.7) we therewith find that for all $j \in \overline{1, k}$

$$
L_{\frac{d}{d t_{j}}}\langle\operatorname{grad} \mathcal{L}[u], d u\rangle=-\frac{d}{d x}\left(d h_{j}[u]+i_{\frac{d}{d t_{1}}} \Omega^{(2)}\right)+d g_{j}(\operatorname{grad} \mathcal{L}[u])
$$


If we put now $u \in M_{N}$, that is $\operatorname{grad} \mathcal{L}[u]=0$, we immediately will find the following: for all $j \in \overline{1, k}$,

$$
d h_{j}[u]+i_{\frac{d}{d t_{j}}} \Omega^{(2)}=0
$$

whence we make a conclusion of the vector field $d / d t_{j}$ to be Hamiltonian on the submanifold $M_{N} \subset M$. Since $d h_{j} / d x=\left\{h^{(x)}, h_{j}\right\}=0, \quad j \in \overline{1, k}$, on the manifold $M_{N}$, we therewith obtain that $d h^{(x)} / d t_{j}=0, j \in \overline{1, k}$. This is also an obvious corollary of the commutativity $\left[d / d t_{j}, d / d x\right]=0, \quad j \in \overline{1, k}$, for all $x, l_{j} \in \mathbb{R}$ on the whole manifold $M$. Indeed, in general case we have the identity $\left\{h^{(x)}, h_{j}\right\}=i_{\left[\frac{d}{d t_{j}}, \frac{d}{d x}\right]} \Omega^{(2)}$, whence the equality $\left\{h^{(x)}, h_{j}\right\} \equiv 0$ on the submanifold $M_{N} \subset M$ takes place immediately, since $\left[\frac{d}{d t_{j}}, \frac{d}{d x}\right]=0$ on $M_{N}$ due to (5.8). The analysis fulfilled above gives a right possibility for a given vector field $d / d t_{j}, \quad j \in \overline{1, k}$, satisfying the either conditions (5.6) on the condition (5.7), to be treated analytically on the canonically symplectic jet-submanifold $M_{N} \subset M$ as a Hamiltonian system.

\section{Liouville integrability}

Now we suppose that the vector field $d / d t_{j}, \quad j \in \overline{1, k}$, all were independent and commutative each with other on the jet-submanifold $M_{N} \subset M$ and with vector fields $d / d t$ and $d / d x$ on the manifold $M$. Besides the submanifold $M_{N} \subset M$ is assumed to be compact one smoothly imbedded into the jet-manifold $J^{(\infty)}\left(\mathbb{R} ; \mathbb{R}^{m}\right)$. If the dimension $\operatorname{dim} M_{N}=2 k+4$, due to the Liouville theorem $[8,9]$ the dynamical systems $d / d x$ and $d / d t$ are Hamiltonian and integrable in quadratures on the submanifold $M_{N} \subset M$. This is the case for all Lax-integrable nonlinear dynamical systems of the Korteweg-de Vries type $[3,7,9,8]$ on functional manifolds.

\section{Discrete dynamical systems. One generalization}

Let us be given a differential discrete smooth dynamical system

$$
d u_{n} / d t=K_{n}[u]
$$

with respect to a continuous evolution parameter $t \in \mathbb{R}$ on the infinitedimensional discrete manifold $M \subset L_{2}\left(\mathbb{Z} ; \mathbb{R}^{m}\right)$ infinite vector-sequences under the condition of rapid decrease in $n \in \mathbb{Z}: \sup _{n \in \mathbb{Z}}|n|^{k}\left\|u_{n}\right\|_{\mathbb{R}^{m}}<\infty$ for all $k \in \mathbb{Z}_{+}$at each point $u=\left(\ldots, u_{n}, u_{n+1}, \ldots\right) \in M$, where $u_{n} \in \mathbb{R}^{m}, \quad n \in \mathbb{Z}$.

Assume further that the dynamical system (7.1) possesses a conservation law $\mathcal{L} \in D(M)$, that is $d \mathcal{L} / d t=0$ along the orbits of (7.1). Via the standard operational approach from (3.5) we can define the variational derivative of $\mathcal{L}:=\sum_{n \in \mathbb{Z}} \mathcal{L}_{n}[u]:$

$$
\operatorname{grad} \mathcal{L}_{n}:=\frac{\delta \mathcal{L}[u]}{\delta u_{n}}=\mathcal{L}_{n}^{*}{ }^{*}[u] \cdot 1
$$

where the last right-hand operation of the multiplying by unity is to be fulfilled by component. 
Lemma 2. Let $\Lambda(M)$ be the infinite-dimensional Grassmannian algebra on the manifold $M$; then the differential $d \mathcal{L}_{n}[u] \in \Lambda^{1}(M)$ concedes the following reduced representation:

$$
d \mathcal{L}_{n}[u]=\left\langle\operatorname{grad} \mathcal{L}_{n}, d u_{n}\right\rangle+d / d n \alpha_{n}^{(1)}[u],
$$

where the one-form $\alpha_{n}^{(1)}[u] \in \Lambda^{1}(M)$ is determined in a unique way, $\langle\cdot, \cdot\rangle$ the scalar product in $\mathbb{R}^{m}$ and $d / d n=\Delta-1, \Delta$-the usual shift operator.

Proof. By definition we obtain for the external differential $d \mathcal{L}_{n}[u]$ the following chain of representations for each $n \in \mathbb{Z}$ :

$$
\begin{aligned}
& d \mathcal{L}_{n}[u]=\sum_{k=0}^{N}\left\langle\frac{\partial \mathcal{L}_{n}[u]}{\partial u_{n+k}}, d u_{n+k}\right\rangle= \\
& =\sum_{k=0}^{N} \sum_{s=0}^{k} \frac{d}{d n}\left(\left\langle\frac{\partial \mathcal{L}_{n-s}[u]}{\partial u_{n+k-s}}, d u_{n+k-s}\right\rangle\right)+\sum_{k=0}^{N}\left\langle\frac{\partial \mathcal{L}_{n-k}[u]}{\partial u_{n}}, d u_{n}\right\rangle \\
& =\frac{d}{d n} \sum_{k=0}^{N} \sum_{s=0}^{k}\left\langle\frac{\partial \mathcal{L}_{n-s}[u]}{\partial u_{n+k-s}}, d u_{n+k-s}\right\rangle+\left\langle\sum_{k=-N}^{N} \Delta^{-k}\left(\frac{\partial \mathcal{L}_{n}[u]}{\partial u_{n+k}}\right), d u_{n}\right\rangle \\
& =\frac{d}{d n} \sum_{k=0}^{N} \sum_{s=0}^{k}\left\langle\frac{\partial \mathcal{L}_{n-s}[u]}{\partial u_{n+k-s}}, d u_{n+k-s}\right\rangle+\left\langle\mathcal{L}_{n}^{\prime *} \cdot 1, d u_{n}\right\rangle \\
& =\frac{d}{d n} \alpha_{n}^{(1)}[u]+\left\langle g r a d \mathcal{L}_{n}, d u_{n}\right\rangle .
\end{aligned}
$$

where $N \in \mathbb{Z}_{+}$- a fixed number depending on the functional $\mathcal{L} \in D(M)$,

$$
\begin{gathered}
\alpha_{n}^{(1)}[u]=\sum_{k=0}^{N} \sum_{s=0}^{k}\left\langle\frac{\partial \mathcal{L}_{n-s}[u]}{\partial u_{n+k-s}}, d u_{n+k-s}\right\rangle \\
=\sum_{k=0}^{N} \sum_{j=0}^{k}\left\langle\frac{\partial \mathcal{L}_{n+j-k}[u]}{\partial u_{n+j}}, d u_{n+j}\right\rangle, \\
\operatorname{grad} \mathcal{L}_{n}=\mathcal{L}_{n}^{\prime *} \cdot 1=\sum_{k=0}^{N} \frac{\partial \mathcal{L}_{n-k}[u]}{\partial u_{n}} .
\end{gathered}
$$

The latter equality in (7.4) proves the lemma 2 completely.

The above proved representation ( 7.3$)$ gives rise to the following stationary problem being posed on the manifold $M$ :

$$
M_{N}=\left\{u \in M: \operatorname{grad} \mathcal{L}_{n}=0\right\}
$$

for' all $n \in \mathbb{Z}$, where by definition det $\left\|\frac{\partial^{2} \mathcal{L} n[u]}{\partial u_{N+1} \partial u_{N+1}}\right\|=0$. In virtue of $(7.3)$ we obtain the validity of the following theorem.

Theorem 3. The finite-dimensional Lagrangian submanifold $M_{N} \subset M$ defined by (7.6), is a symplectic one with the canonical symplectic structure $\Omega_{n}^{(2)}=d \alpha_{n}^{(1)}$ being independent of the discrete variable $n \in \mathbb{Z}$

Proof. From (7.3) we have that on the manifold $M_{N} \subset M \quad d \mathcal{L}_{n}[u]=$ $d / d n\left(\alpha_{n}^{(1)}[u]\right)$, whence for all $n \in \mathbb{Z} \quad d / d n\left(\Omega_{n}^{(2)}\right)=0$. This means obviously, 
that $\Omega_{n+1}^{(2)}=\Omega_{n}^{(2)}$ for all $n \in \mathbb{Z}$, or equivalently, the 2-form $\Omega_{n}^{(2)}$ is not depending on the discrete variable $n \in \mathbb{Z}$. As the 2 -form $\Omega_{n}^{(2)}:=d \alpha_{n}^{(1)}$ by definition, this form is chosen to be a symplectic form on the manifold $M_{N} \subset M$. For this 2 -form which is nondegenerate on $M_{N}$, we assume that Hessian of $\mathcal{L}_{n}$ equals $\operatorname{det}\left\|\frac{\partial^{3} \mathcal{L}_{n}[u]}{\partial u_{n+N+1} \partial u_{n+N+1}}\right\| \neq 0$ on $M_{N}$. The latter proves the theorem.

Let us consider now the prior given dynamical system (7.1) reduced on the manifold $M_{N} \subset M$. To term it as the vector field $d / d t$ on $M_{N}$, we need preliminary to represent it as a Hamiltonian flow on $M_{N}$. To do this, let us write the following identities on $M$ :

$$
\begin{aligned}
i_{\frac{d}{d t}} d\left\langle\operatorname{grad} \mathcal{L}_{n}, d u_{n}\right\rangle & =-\frac{d}{d n} i_{\frac{d}{d t}} \Omega_{n}^{(2)}[u], \\
d i_{\frac{d}{d t}} d\left\langle\operatorname{grad} \mathcal{L}_{n}, d u_{n}\right\rangle & =-\frac{d}{d n}\left(d h_{n}^{(t)}[u]\right),
\end{aligned}
$$

which are valid for all $n \in \mathbb{Z}$. Adding the last identities in (7.7), we come to the following one for all $n \in \mathbb{Z}$ :

$$
\frac{d}{d t}\left\langle\operatorname{grad} \mathcal{L}_{n}, d u_{n}\right\rangle=-\frac{d}{d n}\left(i_{\frac{d}{d t}} \Omega_{n}^{(2)}[u]+h_{n}^{(t)}[u]\right) .
$$

Having reduced the identity (37) upon the manifold $M_{N} \subset M$, we obtain the wished expression for all $u \in M_{N}, \quad N \in \mathbb{Z}$ :

$$
i_{\frac{d}{d t}} \Omega_{n}^{(2)}[u]+h_{n}^{(t)}[u]=0
$$

The latter means that the dynamical system (7.1) on the manifold $M_{N}$ is a Hamiltonian one, the function $h_{n}^{(t)}[u]$ being a Hamiltonian function defined explicitly by the second identity in (7.7).

We assume now that the symplectic structure $\Omega_{n}^{(2)}[u]$ on $M_{N}$ be repre; sentable as follows:

$$
\Omega_{n}^{(2)}[u]=\sum_{j=0}^{N}\left\langle d p_{j+n} \wedge d u_{j+n}\right\rangle
$$

where generalized impulses $p_{j+n} \in \mathbb{R}^{m}, \quad j=\overline{0, N}$, are determined from the following identifications: $\alpha_{n}[u]:=\mathcal{L}\left(u_{n}, u_{n+1}, \ldots, u_{n+N+1}\right) \in \mathbb{R}, \quad n \in \mathbb{Z}$,

$$
\begin{aligned}
\alpha_{n}[u]: & =\sum_{j=0}^{N}\left\langle d p_{j+n}, d u_{j+n}\right\rangle=\sum_{k=0}^{N} \sum_{j=0}^{k}\left\langle\frac{\partial \mathcal{L}_{n+j-k}[u]}{\partial u_{n+j}}, d u_{n+j}\right\rangle= \\
& =\sum_{j=0}^{N} \sum_{k=j}^{N}\left\langle\frac{\partial \mathcal{L}_{n+j-k}[u]}{\partial u_{n+j}}, d u_{n+j}\right\rangle
\end{aligned}
$$

whence we get the final expression:

$$
p_{j+n}:=\sum_{k=j}^{N} \frac{\partial \mathcal{L}_{n+j-k}[u]}{\partial u_{n+j}}
$$


where $j=\overline{0, N}, \quad u \in M_{N} \subset M$.

Now we are in position to formulate the given dynamical system (7.1) as that on the reductive manifold $M_{N} \subset M$ :

$$
\begin{aligned}
& \frac{d u_{n+j}}{d t}=\left\{h_{n}^{(t)}, u_{n+j}\right\}=\frac{\partial h_{n}^{(t)}}{\partial p_{n+j}} \\
& \frac{d p_{n+j}}{d t}=\left\{h_{n}^{(t)}, p_{n+j}\right\}=-\frac{\partial h_{n}^{(t)}}{\partial u_{n+j}}
\end{aligned}
$$

for all $n \in \mathbb{Z},, j=\overline{0, N}$. Thereby the problem of embedding the given discrete dynamical system (7.1) into a vector field flow on the manifold $M_{N} \subset M$ is solved completely with the final result (7.13).

\section{Invariant Lagrangian construction (functional manifold case)}

In the case when the given nonlinear dynamical system (2.1) is integrable one of Lax-type, we can proceed effectively to find a commutive infinite hierarchy of conservation laws can be serving as the invariant Lagrangians, under considered above.

At first we have to use the important property [3] of the gradient functional $\varphi=\operatorname{grad} \gamma \in T^{*}(M)$ generated by an arbitrary conservation law $\gamma \in D(M)$, i.e. the following Lax-type equation :

$$
d \varphi / d t+K^{\prime *} \varphi=0
$$

where the prime sign denotes the usual Frechet derivative of the local functional $K: M \rightarrow T(M)$ on the manifold $M$, the star "*" denotes its conjugation operator with respect to the nondegenerate standard convolution functional $(\cdot, \cdot)=\int_{\mathbb{R}} d x\langle\cdot, \cdot\rangle$ on $T^{*}(M) \times T(M)$. The equation (8.1) admits, what follows from [30-32], the special asymptotical kind of solution:

$$
\varphi(x, t ; \lambda) \cong(1, a(x, t ; \lambda))^{\tau} \exp \left[\omega(x, t, \lambda)+\partial^{-1} \sigma(x, t ; \lambda)\right],
$$

where $a(x, t ; \lambda) \in \mathbb{R}^{m-1}, \quad \sigma(x, t ; \lambda) \in \mathbb{R}, \quad \omega(x, t ; \lambda)$-some dispersive function. The sign " $\tau$ " means here the transposition one, what is adopted in matrix analysis. For any complex parameter $\lambda \in \mathbb{C}$ at $|\lambda| \rightarrow \infty$ the following expansions take place:

$$
\begin{aligned}
& a(x, t ; \lambda) \simeq \sum_{j \in \mathbb{Z}_{+}} a_{j}[x, t ; u] \lambda^{-j+s(a)}, \\
& \sigma(x, t ; \lambda) \simeq \sum_{j \in \mathbb{Z}_{+}} \sigma_{j}[x, t ; u] \lambda^{-j+s(\sigma)} .
\end{aligned}
$$

Here $s(a)$ and $s(\sigma) \in \mathbb{Z}_{+}$- some appropriate nonnegative integers, the operation $\partial^{-1}$ means the inverse one to the differentiation $d / d x$, that is $d / d x \cdot \partial^{-1}=1$ for all $x \in \mathbb{R}$.

To find the explicit form of the representation (8.2) in the case when the associated Lax-type representation [8] depends parametrically on the 
spectral parameter $\lambda(t ; \lambda) \in \mathbb{C}$, satisfying the following nonisospectral condition:

$$
d \lambda(t ; \lambda) / d t=g(t ; \lambda(t ; \lambda)),\left.\quad \lambda(t ; \lambda)\right|_{t=0}=\lambda \in \mathbb{C},
$$

for some meromorphic function $g(t ; \cdot): \mathbb{C} \rightarrow \mathbb{C}, t \in \mathbb{R}_{+}$, we must reanalyse more carefully the asymptotic solutions to the Lax equation (8.1). Namely, we are going to treat more exactly the case when the solution $\varphi \in T^{*}(M)$ to $(8.1)$ is represented as an appropriate trace-functional of a Lax spectral problem at the moment $\tau=t \in \mathbb{R}_{+}$with the spectral parameter $\lambda(t ; \lambda) \in \mathbb{C}$ satisfying the condition (8.3), the evolution of the given dynamical system (2.1) being considered with respect to the introduced above parameter $\tau \in$ $\mathbb{R}$, that is

$$
d u / d \tau=K[x, \tau ; u],
$$

$\left.u\right|_{\tau=0}=\bar{u} \in M-$ some Canchy data of $M$. This means that the functional

$$
\tilde{\varphi}(x, \tau ; \tilde{\lambda}):=\operatorname{grad} \operatorname{Sp} S(x, \tau ; \tilde{\lambda}), \quad \tilde{\lambda}=\tilde{\lambda}(\tau ; \lambda(t ; \lambda)) \in \mathbb{C},
$$

has to satisfy the corresponding Lax equation at the point $u \in M$ subject to $(8.4)$ :

$$
d \tilde{\varphi} / d \tau+K^{\prime *}[u] \cdot \tilde{\varphi}=0
$$

for all $\tau \in \mathbb{R}_{+}$. Under the above assumption it is obvious that the spectral parameter $\tilde{\lambda}=\tilde{\lambda}(\tau ; \lambda(t ; \lambda))$, where

$$
d \tilde{\lambda} / d \tau=\tilde{g}(\tau ; \tilde{\lambda}),\left.\quad \tilde{\lambda}\right|_{\tau=0}=\lambda(t ; \lambda) \in \mathbb{C},
$$

$\tilde{g}(t ; \cdot): \mathbb{C} \rightarrow \mathbb{C}-$ some meromorphic function being found simply from (8.6), the Cauchy data $\lambda(t ; \lambda) \in \mathbb{C}$ for all $t \in \mathbb{R}_{+}$are the corresponding ones to (8.3), the parameter $\lambda \in \mathbb{C}$ being a spectrum value of the associate Lax type spectral problem at the moment $t \in \mathbb{R}_{+}$.

Now we are about to formulate the following lemma.

Lemma 3. The Lax equation (8.6) at, the parameter $\tau \rightarrow t \in \mathbb{R}_{+}$admits the asymptotical solution

$$
\tilde{\varphi}(x, \tau ; \tilde{\lambda}) \cong(1, \tilde{a}(x, \tau ; \tilde{\lambda}))^{\tau} \exp \left[\tilde{\omega}(x, \tau, \tilde{\lambda})+\partial^{-1} \tilde{\sigma}(x, \tau ; \tilde{\lambda})\right],
$$

where $\tilde{a}(x, \tau ; \tilde{\lambda}) \in \mathbb{R}^{m-1}, \quad \tilde{\sigma}(x, \tau ; \tilde{\lambda}) \in \mathbb{R},-$ some local functionals on $M$, $\tilde{\omega}(x, \tau, \tilde{\lambda}) \in \mathbb{R}$ - some discrete function for all $x \in \mathbb{R}, \tau \in \mathbb{R}_{+}$, and if for $|\lambda| \rightarrow$ $\infty$ the property $|\tilde{\lambda}| \rightarrow \infty$ at $\tau \rightarrow t \in \mathbb{R}_{+}$holds, the following expansions are valid:

$$
\begin{aligned}
& \tilde{a}(x, \tau ; \tilde{\lambda}) \simeq \sum_{j \in \mathbb{Z}_{+}} \tilde{a}_{j}[x, \tau ; u] \tilde{\lambda}^{-j+s(\tilde{a})}, \\
& \tilde{\sigma}(x, \tau ; \tilde{\lambda}) \simeq \sum_{j \in \mathbb{Z}_{+}} \tilde{\sigma}_{j}[x, \tau ; u] \tilde{\lambda}^{-j+s(\tilde{\sigma})},
\end{aligned}
$$

where $s(\tilde{a})$ and $s(\tilde{\sigma}) \in \mathbb{Z}_{+}$are some integers.

Proof. Due to the theory of asymptotic expansions for arbitrary differential spectral problems, the result $(8.8)$ will hold provided the representation (8.5) is valid and the spectral parameter $\lambda(t ; \lambda)$ is not depending on $\tau \in \mathbb{R}_{+}$ effectively at the moment $\tau \rightarrow t \in \mathbb{R}_{+}$, when it subjects to (8.7). But the 
above situation is occasional because of the Lax-type integrability of the dynamical system (8.4). Indeed, due to the integrability of (8.4), the spectral parameter $\tilde{\lambda} \in \mathbb{C}$ is matching the equation (8.7), and at the moment $\tau \rightarrow t \in \mathbb{R}_{+}$the Cauchy data $\lambda(t ; \lambda) \in \mathbb{C}$ no longer depend on the parameter $\tau \in \mathbb{R}_{+}$effectively, that is at $\tau \rightarrow t \in \mathbb{R}_{+}$the condition $d \lambda(x, \lambda) / d \tau \rightarrow 0$ is valid. The latter proves the lemma completely.

As a result of the Lemma 3 we can formulate now the following important theorem.

Theorem 4. The Lax integrable parametrically isospectral dynamical system (8.4) at $\tau \rightarrow t \in \mathbb{R}_{+}$admits an infinite hierarchy of conservation laws, in general nonuniform ones with respect to the variables $x \in \mathbb{R}, \tau \in$ $\mathbb{R}_{+}$, which can be represented in an exact form in virtue of the asymptotic expansion (8.8) and (8.9).

Proof.Indeed, due to the expansion (8.8), we can obtain right away that the functional

$$
\tilde{\gamma}(\tau ; \lambda(t ; \lambda))=\int_{\mathbb{R}} d x \tilde{\sigma}(x, \tau ; \tilde{\lambda}(\tau ; \lambda(t ; \lambda)))
$$

does not depend at $\tau \rightarrow t \in \mathbb{R}_{+}$on the parameter $\tau \in \mathbb{R}_{+}$, that is

$$
d \tilde{\gamma} /\left.d \tau\right|_{\tau \rightarrow t \in \mathbb{R}_{+}}=0
$$

for all $t \in \mathbb{R}_{+}$. If we make also the parameter $\tau \in \mathbb{R}_{+}$to tend to $t \in \mathbb{R}_{+}$, due to (8.5) we obtain that $\left.\tilde{\varphi}(x, \tau ; \tilde{\lambda})\right|_{\tau \rightarrow t \in \mathbb{R}_{+}} \rightarrow \varphi(x, t ; \lambda)$ for all $x \in \mathbb{R}, t \in \mathbb{R}_{+}$ and $\lambda \in \mathbb{C}$. This means that the local functional $\varphi(x, t ; \lambda) \in T^{*}(M)$ satisfies the equation (8.1) at each point $u \in M$. As the obvious result, the following identifications hold:

$$
\begin{gathered}
\left.\tilde{\omega}(x, \tau ; \tilde{\lambda})\right|_{\tau \rightarrow t \in \mathbb{R}_{+}} \rightarrow \omega(x, t ; \lambda) \\
\left.\tilde{\sigma}(x, \tau ; \tilde{\lambda})\right|_{\tau \rightarrow t \in \mathbb{R}_{+}} \rightarrow \sigma(x, t ; \lambda)
\end{gathered}
$$

for all $\lambda \in \mathbb{C}$. Hence, the functional $\gamma(\lambda):=\left.\tilde{\gamma}(\tau ; \lambda(t ; \lambda))\right|_{\tau \rightarrow t \in \mathbb{R}_{+}}=\int_{\mathbb{R}} d x \sigma(x, t ; \lambda) \in D(M)$ doesn't depend on the evolution parameter $t \in \mathbb{R}_{+}$and due to equation (8.1), it is a conserved quantity for the nonlinear dynamical system. (2.1) under consideration, i.e.

$$
d \gamma(t ; \lambda) / d t=0
$$

for all $t \in \mathbb{R}_{+}$and $\lambda \in \mathbb{C}$. Therefore, we have a possibility to use the equation (8.12) along with (8.7) for the asymptotic expansions (8.9) and (8.3) to be found in exact form. To do this we at first need to insert the asymptotic expansion (8.8) in the determining equation (8.6) for the asymptotic expansions (8.9) to be searched explicitely at the moment $\tau \rightarrow$ $t \in \mathbb{R}_{+}$. Keeping in mind that at $\tau \rightarrow t \in \mathbb{R}_{+}|\lambda| \rightarrow \infty$ if $|\tilde{\lambda}| \rightarrow \infty$, and solving step by step the resulting recurrence relationships for the coefficients in (8.9), we will get the functional $\gamma(\lambda):=\left.\tilde{\gamma}(\tau ; \lambda(t ; \lambda))\right|_{\tau \rightarrow t \in \mathbb{R}_{+}}, \quad \lambda \in \mathbb{C}$, in form appropriate for the criterial equation (8.12) to be used. As the second step, we need to employ the differential equation ( 8.7$)$ for the criterial 
equation (8.12) to be satisfied point-wise for all $t \in \mathbb{R}_{+}$. This means, in particular, that

$$
\begin{gathered}
\frac{d \gamma(\lambda)}{d t}=\left.\frac{d}{d t} \sum_{j \in \mathbb{Z}_{+}} \int_{\mathbb{R}} d x \tilde{\sigma}_{j}[x, \tau ; u] \tilde{\lambda}^{-j+s(\tilde{\sigma})}\right|_{\tau=t \in \mathbb{R}_{+}} \\
=\left.\int_{\mathbb{R}} d x \sum_{j \in \mathbb{Z}_{+}}\left[\frac{d \tilde{\sigma}_{j}[x, \tau ; u]}{d t} \tilde{\lambda}^{-j+s(\tilde{\sigma})}+\tilde{\sigma}_{j}[x, \tau ; u] \tilde{\lambda}^{-j+s(\tilde{\sigma})-1}(s(\tilde{\sigma})-j) \frac{d \tilde{\lambda}}{d t}\right]\right|_{\tau=t \in \mathbb{R}_{+}} \\
\Rightarrow \int_{\mathbb{R}} d x \sum_{j \in \mathbb{Z}_{+}}\left[\left(d \tilde{\sigma}_{j} / d t\right) \tilde{\lambda}^{-j+s(\tilde{\sigma})}+\sum_{k>>-\infty}(s(\tilde{\sigma})-k) \tilde{\sigma}_{k} \tilde{g}_{j-k-1}(t) \tilde{\lambda}^{-j+s(\tilde{\sigma})}+\right. \\
\left.+\sum_{j \in \mathbb{Z}_{+}} \tilde{\sigma}_{j}[x, t ; u] \tilde{\lambda}^{-j+s(\tilde{\sigma})-1}(s(\tilde{\sigma})-j) \frac{\partial \tilde{\lambda}}{\partial \lambda} g(t ; \lambda)\right] \equiv 0,
\end{gathered}
$$

where we put by definition $\tilde{g}(\tau ; \tilde{\lambda}): \simeq \sum_{k \gg>-\infty} \tilde{g}_{k}(\tau) \tilde{\lambda}^{-k}$ for all $\tau \in \mathbb{R}_{+}$and $|\lambda| \rightarrow \infty$. Since the spectral parameter $\tilde{\lambda}=\tilde{\lambda}(t ; \lambda)$ at the moment $t=0$ coincides with an arbitrary complex value $\lambda \in \mathbb{C}$, the condition $|\lambda| \rightarrow \infty$ together with (8.13) at the moment $t=0$ gives rise to the following recurrent relationships:

$$
\begin{gathered}
\sum_{j \in \mathbb{Z}_{+}}\left[\partial \tilde{\sigma}_{j} / d t+\tilde{\sigma}_{j}^{\prime} \cdot K[t ; u]+\sum_{k>>-\infty}(s(\tilde{\sigma})-k) \tilde{\sigma}_{k} \cdot \tilde{g}_{j-k-1}\right] \tilde{\lambda}^{-j+s(\tilde{\sigma})}= \\
\quad=\sum_{j \in \mathbb{Z}_{+}} \tilde{\sigma}_{j}(s(\tilde{\sigma})-j) \frac{\partial \tilde{\lambda}}{\partial \lambda} g(t ; \lambda) \tilde{\lambda}^{-j+s(\tilde{\sigma})-1} \equiv 0: \bmod (d / d x)
\end{gathered}
$$

for all $j \in \mathbb{Z}_{+}, x \in \mathbb{R}, t \in \mathbb{R}_{+}$and $u \in M$. Having solved the algebraic relationships (8.14) for the prior unknown function $g(t), t \in \mathbb{R}_{+}$, we will obtain the genereting functional $\gamma(\lambda), \lambda \in \mathbb{C}$, of conservation laws for $(2.1)$ in exact form. This completes the constructive part of the proof of the theorem above.

For a practical use we need obviously to solve further the differential equation (8.3) at the moment $\tau \rightarrow t \in \mathbb{R}_{+}$in exact, maybe in asymptotic form for the dispersive function $\omega(x, t ; \lambda)$ and the local generative functional $\sigma(x, t ; \lambda)$ to be determined via (8.11) exactly for all $x \in \mathbb{R}, t \in \mathbb{R}_{+}$and $|\lambda| \rightarrow$ $\infty$. This note together with the possibility to apply the general scheme of the gradient-holonomic algorithm gives rise to determining the above mentioned Lax-type representation completely in exact form, what successfully solves the rather complex direct problem of the integrability theory of nonlinear dynamical systems on functional manifolds.

Having obtained the generation function $\gamma(\lambda) \in D(M), \lambda \in \mathbb{C}$, of an infinite hierarchy of conservation laws of the dynamical system (2.1) on the manifold $M$, we can build appropriately a general Lagrangian functional $\mathcal{L}_{N} \in D(M)$ as follows:

$$
\mathcal{L}_{N}=-\gamma_{N+1}+\sum_{j=0}^{N} c_{j} \gamma_{j}
$$


where, by definition, $\gamma(\lambda)=\int_{\mathbb{R}} d x \sigma(x, t ; \lambda)$ and for $|\lambda| \rightarrow \infty$ functionals $\gamma_{j}=\int_{\mathbb{R}} d x \sigma_{j}[x, t ; \lambda], j \in \mathbb{Z}_{+}$, are conservation laws due to expansion (8.2), $c_{j} \in \mathbb{R}, j=\overline{0, N}$ - some arbitrary constants, $N \in \mathbb{Z}_{+}$- an arbitrary nonnegative integer. If the differential order of the functional $\gamma_{N+1} \in D(M)$ has the highest one of the orders of functionals $\gamma_{j} \in D(M), j=\overline{0, N}$, and additionally, this Lagrangian is not degenerate, that is $\operatorname{det}\left(\right.$ Hess $\left.\gamma_{N+1}\right) \neq 0$, we can apply all the theory developed before, to prove the critical submanifold $M_{N}=\left\{u \in M: \operatorname{grad} \mathcal{L}_{N}=0\right\}$ to be the finite-dimensional symplectic manifold inserted into the standard jet-manifold $J^{(\infty)}\left(\mathbb{R} ; \mathbb{R}^{m}\right)$ with the canonical symplectic structure subject to the vector field $d / d x, x \in \mathbb{R}$, being the Hamiltonian flow on the submanifold $M_{N}$.

\section{Invariant Lagrangian construction (discrete manifold case)}

Let us consider the discrete Lax integrable dynamical system on the discrete manifold $M$ without an apriory given Lax-type representation. The problem arises how to get the corresponding conservation laws via the gradientholonomic algorithm [8]. To realise this way let us study solutions to the Lax equation:

$$
d \varphi_{n} / \dot{d}+K_{n}^{\prime}[\tau, u] \cdot \varphi_{n}=0
$$

local functionals $\varphi_{n}[u] \in T_{u_{n}}^{*}(M)$ at the point $u_{n} \in M, n \in \mathbb{Z}$. By analogy with the approach of Chapter 7 we assert that equation (9.1) admits a generating solution $\varphi_{n}=\varphi_{n}(t ; \lambda) \in T_{u_{n}}^{*}(M), n \in \mathbb{Z}, \lambda \in \mathbb{C}-$ a complex parameter in the form:

$$
\varphi_{n} \cong\left(1, a_{n}(t ; \lambda)\right)^{\tau} \exp [\omega(t ; \lambda)]\left(\prod_{j=-\infty}^{n} \sigma_{j}(t ; \lambda)\right),
$$

where $\omega(t ; \lambda)$ - some dispersive function for $t \in \mathbb{R}_{+}, \quad a_{n}(t ; \lambda) \in \mathbb{R}^{m-1}$, $\sigma_{n}(t ; \lambda) \in \mathbb{R}$ - local functionals on $M$, having the following asymptotic expansions at $|\lambda| \rightarrow \infty$ :

$$
\begin{aligned}
a_{n}(t ; \lambda) & \simeq \sum_{j \in \mathbb{Z}_{+}} a_{n}[t ; u] \lambda^{-j+s(a)}, \\
\sigma_{n}^{(j)}(t ; \lambda) & \simeq \sum_{j \in \mathbb{Z}_{+}} \sigma_{n}[t ; u] \lambda^{-j+s(\sigma)} .
\end{aligned}
$$

To find the explicit form of the asymptotic representation $(9.2)$ we need to study additionally the asymptotic solutions to the following attached Lax equation with respect to the new external parameter $\tau \in \mathbb{R}_{+}$:

$$
d \tilde{\varphi}_{n} / d \tau+K_{n}^{\prime *}[\tau, u] \cdot \tilde{\varphi}_{n}=0
$$

where $\tilde{\varphi}_{n} \in T^{*}(M)$, a point $u \in M$ is subject to the following dynamical system:

$$
d u_{n} / d \tau=K_{n}[\tau ; u], \quad n \in \mathbb{Z} .
$$

Having made the assumption above we can assert that equation (9.4) admits the asymptotic solution in the form:

$$
\tilde{\varphi}_{n}(\tau ; \tilde{\lambda}) \cong\left(1, \tilde{a}_{n}(\tau ; \tilde{\lambda})\right)^{\tau} \exp [\tilde{\omega}(\tau ; \tilde{\lambda})] \prod_{j=-\infty}^{n} \tilde{\sigma}_{j}(\tau ; \tilde{\lambda}),
$$


where for all $n \in \mathbb{Z}$ at $\tau \rightarrow t \in \mathbb{R}_{+}$

$$
\begin{aligned}
& \tilde{a}_{n}(\tau ; \tilde{\lambda}) \simeq \sum_{j \in \mathbb{Z}_{+}} \tilde{a}_{n}^{(j)}[x, \tau ; u] \tilde{\lambda}^{-j+s(\tilde{a})}, \\
& \tilde{\sigma}_{n}(\tau ; \tilde{\lambda}) \simeq \sum_{j \in \mathbb{Z}_{+}} \tilde{\sigma}_{n}^{(j)}[\tau ; u] \tilde{\lambda}^{-j+s(\tilde{\sigma})} .
\end{aligned}
$$

The expansions above are valid if $|\tilde{\lambda}| \rightarrow \infty$ at $|\lambda| \rightarrow \infty$ and $d \lambda(t ; \lambda) /\left.d \tau\right|_{\tau \rightarrow t \in \mathbb{R}_{+}}$ $\rightarrow 0$ for all $t \in \mathbb{R}_{+}$. The latter case is available because of the Lax-integra$\overrightarrow{b i l i t y}$ of the dynamical system (9.5). This means that a spectrum evolution

$$
d \tilde{\lambda} / d \tau=\tilde{g}(\tau ; \tilde{\lambda}),\left.\quad \tilde{\lambda}\right|_{t=0}=\lambda(t ; \lambda) \in \mathbb{C},
$$

for an associated Lax-type representation to the equation $(9.5)$, where $g(\tau ; \cdot)$ : $\mathbb{C} \rightarrow \mathbb{C}$-some meromorphic mapping for all $t \in \mathbb{R}_{+}$, satisfies the integrability condition $\left.\lambda(\tau)\right|_{\tau \rightarrow t \in \mathbb{R}_{+}} \rightarrow \lambda \in \mathbb{C}$, whence the expression $\left.\frac{d \lambda(t ; \lambda)}{d \tau}\right|_{\tau \rightarrow t \in \mathbb{R}_{+}} \rightarrow$ 0 holds for all $t \in \mathbb{R}_{+}$.

Substituting the expansions (9.6) and (9.7) into (9.4), we obtain some recurrence relationships, enabling to find local functionals $\tilde{\sigma}_{j}[t ; u], j \in \mathbb{Z}_{+}$, to be found exactly. Having this done succesefully, we assert that the functional

$$
\gamma(t ; \lambda)=\left.\sum_{n \in \mathbb{Z}} \ln \tilde{\sigma}_{n}(\tau ; \tilde{\lambda})\right|_{\tau \rightarrow t \in \mathbb{R}_{+}} \equiv \sum_{n \in \mathbb{Z}} \ln \sigma_{n}(t ; \lambda),
$$

where $\tilde{\lambda}=\tilde{\lambda}(\tau ; \lambda), \tau \in \mathbb{R}_{+}, \lambda \in \mathbb{C}$, - the meromorphic solution to the equation (9.8). To find further the exact form of the equation (9.8), we need only to find an evolution $\frac{d \lambda}{d t}=g(t ; \lambda)$, recalling that the local functional. $\left.\tilde{\varphi}_{n}(\tau ; \tilde{\lambda})\right|_{\tau \rightarrow t \in \mathbb{R}_{+}} \rightarrow \varphi_{n}(t ; \lambda) \in T^{*}(M)$ for all $t \in \mathbb{R}_{+}$and $\lambda \in \mathbb{C}$. Hence, the following equality holds immediately:

$$
\begin{gathered}
\left.\frac{d}{d t} \sum_{n \in \mathbb{Z}_{+}} \ln \tilde{\sigma}_{n}(\tau ; \tilde{\lambda})\right|_{\tau \rightarrow t \in \mathbb{R}_{+}}= \\
\sum_{n \in \mathbb{Z}_{+}} \tilde{\sigma}_{n}^{-1}(t ; \tilde{\lambda})\left[\frac{\partial \tilde{\sigma}_{n}}{\partial t}+\tilde{\sigma}_{n}^{\prime} \cdot K_{n}[u]+\frac{\partial \tilde{\sigma}_{n}}{\partial \tilde{\lambda}} g(t ; \tilde{\lambda})+\right. \\
\left.+\frac{\partial \tilde{\sigma}_{n}}{\partial \tilde{\lambda}} \frac{\partial \tilde{\lambda}}{\partial \lambda} g(t ; \lambda)\right]=0
\end{gathered}
$$

for all $t \in \mathbb{R}_{+}$. Equating coefficients of $(9.10)$ at all powers of the spectral parameter $\lambda \in \mathbb{C}$ to zero modulus $d / d n, n \in \mathbb{Z}$, we will find the recurrent relationships for the function $\tilde{g}(t ; \lambda)$ of $(9.8)$ to be determined successfully. Thereby, using the equation (9.9) and an expansion $\sigma(t ; \lambda) \simeq$ $\sum_{j \in \mathbb{Z}_{+}} \gamma_{j}[t ; u] \lambda^{-j+s(\gamma)}$ for $|\lambda| \rightarrow \infty$, where $s(\gamma) \in \mathbb{Z}_{+}$- some integer number, we obtain an infinite hierarchy of discretewise conservation laws of the initially given nonlinear dynamical system (2.1) on the manifold $M$. But because of the parametric dependence of the conservation laws built above on the evolution parameter $t \in \mathbb{R}_{+}$, we cannot use right now the theory evolved before to prove the Hamiltonian properties of the corresponding vector fields on the invariant submanidolds. To do this in an appropriate way, we are going below to augment the theory of some moments thoroughly. 


\section{Reduction procedure revised}

Below we shall use the results obtained in chapters 8 and 9 , concerning the construction of parametrically invariant Lagrangian submanifols in the phase space $M$ of given nonlinear parametrically integrable dynamical systems. Define an invariant submanifold $M_{N} \subset M$ as follows:

$$
M_{N}:=\left\{u \in M: \operatorname{grad} \mathcal{L}_{N}[x, t ; u]=0\right\}
$$

where the Lagrangian $\mathcal{L}_{N} \in D(M)$ is specified as that in (8.15).

Our next problem is to describe an infinite hierarchy of symmetries of the given dynamical system, i.e. we will be looking for vector fields $d u / d \tau_{j}=$ $\alpha_{j}[x, t ; u], j \in \mathbb{Z}_{+}$, on $M$, for which the expressions

$$
\frac{d \alpha_{j}}{d t}-K^{\prime \prime} \cdot \alpha_{j}=0
$$

hold. As the dynamical systems $\alpha_{j}[x, t ; u], j \in \mathbb{Z}_{+}$, don't depend on the evolution parameter $\tau_{j} \in \mathbb{R}, j \in \mathbb{Z}_{+}$, we may conclude that all vector fields $d / d \tau_{j}, j \in \mathbb{Z}_{+}$, on $M$ are Hamiltonian with respect to implectic operator $\theta$ : $T^{*}(M) \rightarrow T(M)$, satisfying the following operator equation: $\frac{\partial \theta}{\partial t}+L_{K} \theta=0$, or

$$
d \theta / d t-\theta \cdot K^{\prime *}-K^{\prime} \cdot \theta=0 .
$$

Having solved the equation above by means of the method of a small parameter [8], we can build commuting vector fields $d / d \tau_{j}, j \in \mathbb{Z}_{+}$, as
follows:

$$
d u / d \tau_{j}=-\theta \operatorname{grad} \gamma_{j}[x, t ; u]=: \alpha_{j}[x, t ; u],
$$

where $t \in \mathbb{R}_{+}$serves as a parameter.

Indeed, the equation (10.2) holds due to the necessary condition that elements $\varphi_{j}:=\operatorname{grad} \gamma_{j} \in T^{*}(M)$ would match the following relationships: $\partial \varphi_{j} / \partial t+L_{K} \varphi_{j}=0$, or

$$
d \varphi_{j} / d t+K^{\prime *} \cdot \varphi_{j}=0, \quad \varphi_{j}^{*}=\varphi_{j}^{\prime}
$$

for all $j \in \mathbb{Z}_{+}, t \in \mathbb{R}_{+}$. But the latter is obviously true due to the fact that functionals $\gamma_{j} \in D(M), j \in \mathbb{Z}_{+}$, are conservation laws for (2.1). Thereby, we may construct reduced vector fields $d / d \tau_{j}, j \in \mathbb{Z}_{+}$, on the invariant submanifold $M_{N} \subset M$ via the standard way developed before in this article.

To escape perhaps some tedious procedure for the equation (10.3) to be solved in exact form, we may proceed also with the following way: one solves the generalized Lax equation (by means of the method of a small parameter for instance)

$$
\frac{d \varphi}{d t}+K^{\prime * *} \cdot \varphi=\operatorname{grad} \xi
$$

for some fixed but arbitrary functional $\xi \in D(M)$ with the additional condition $\varphi^{\prime} \neq \varphi^{\prime *}$ on $M$. As a result we claim that the operator

$$
\theta^{-1}=\varphi^{\prime *}-\varphi^{\prime}
$$

is a cosymplectic one on the manifold $M$ for all dynamical systems (10.4), that is $L_{\alpha_{j}} \theta=0$ for all $j \in \mathbb{Z}_{+}$. Besides, if we represented the dynamical system $d u / d t=K[x, t ; u]$ on $M$ as $d u / d t=-\eta \varphi[x, t ; u], \quad \eta: T^{*}(M) \rightarrow T(M)$ 
- some implectic on $M$ operator, then, due to (10.7) and to the identity $L_{\eta \varphi} \eta^{-1}=\varphi^{\prime}-\varphi^{\prime *} \Rightarrow-\frac{\partial \eta^{-1}}{\partial t}$, we would obtain the operator $\theta^{-1}: T(M) \rightarrow$ $T^{*}(M)$ rather simply by a differentiation with respect to the independent variable $t \in \mathbb{R}$. Also as a simple consequence, we get the infinite series of equalities: $L_{d / d \tau_{j}} \theta=0, \quad L_{d / d \tau_{j}} \eta=0, j \in \mathbb{Z}_{+}$. In the case when the operator pair $(\theta, \eta)$ is independent, we claim they to compile a compatible pair of implectic operators on $M$. As the latter is the case, we can therefore use the standard gradient-holonomic algorithm [8] for the associated Lax pair to the dynamical system (2.1) to be found explicitely, on what we won't enlarge here.

\section{The reduction procedure on nonlocal Lagrangian sub- manifolds}

Let us consider the following nonusual reduction problem for a given Lax type integrable dynamical system

$$
d u / d t=K[u]
$$

on the manifold $M \ni u$; augmented by the following compatible dynamical eigenfunction relationships:

$$
\begin{aligned}
d f / d t & =p(l) f \\
-d f^{*} / d t & =p^{*}(l) f^{*} .
\end{aligned}
$$

Here, by definition,

$$
l[u] f=\lambda f, \quad l^{*}[u] f^{*}=\lambda f^{*}
$$

where $l[u]$ - some scalar pseudo-differential operator with the symbol $\xi$, a spectral parameter $\lambda \in \mathbb{C}, f, f^{*} \in W(\mathbb{R} ; \mathbb{C})$ - element of some Sobolev type Hilbert space, the Lax pair $l[u ; \lambda]$ and $p(l)$ acts on which. As a result of (11.2) and (11.3) we demand the standard Lax type representation

$$
d l / d t=[l, p(l)]
$$

to hold for all $\lambda \in \mathbb{C}, t \in \mathbb{R}, u \in M$, satisfying the equation (11.1).

We pose the following problem: if we are given the functionals $\gamma_{j}:=$ $\operatorname{tr} l^{j} \in \mathcal{D}(M), j \in Z_{+}, \quad \operatorname{tr}():.=\int_{\mathbb{R}} d x \operatorname{res} s_{\xi=10}($.$) , as local conservation laws$ for (11.1) and functionals $\lambda_{j} \in \mathcal{D}(M), j=\overline{0, N(\lambda)}$ as generalized nonlocal eigenvalue functionals (11.3), i.e. for all $j=\overline{1, N(\lambda)}, f_{j}=f\left(x ; \lambda_{j}\right) \epsilon$ $W(\mathbb{R} ; \mathbb{C})$, how to find the reduced flow of $(11.1)$ on the following apriori invariant submanifold $M_{N}^{\prime} \subset W \times M \times W, M_{N}^{\prime}:=\left\{\left(f, u, f^{*}\right) \in W \times M \times W\right.$ : $\left.\operatorname{grad\mathcal {L}_{N}^{\prime }}[u]=0\right\}$, where

$$
\mathcal{L}_{N}^{\prime}:=\sum_{j=0}^{N(\gamma)} a_{j} \gamma_{j}+\sum_{j=0}^{N(\lambda)} b_{k} \lambda_{k}
$$

$a_{j} \in \mathbb{C}, j=\overline{0, N(\gamma)}$, and $b_{k} \in \mathbb{C}, k=\overline{0, N(\lambda)}$ - some arbitrary but fixed constant complex parameter. 
To build the reduced flow of (11.1) on nonlocal the manifold $M_{N}$, one needs to have the canonical symplectic structure on it, inherited by the functional (11.5). For this aim let us evaluate at first the gradient $\nabla \lambda_{k}\left[f, u, f^{*}\right] \in$ $T^{*}(W \times M \times W), k=\overline{0, N(\lambda)}$, using variations of equations (11.3):

$$
\begin{gathered}
\left(f^{*}, l[u] f\right)_{W}:=\int_{\mathbb{R}^{*}}\left\langle f^{*}, l[u] f\right\rangle d x=\lambda\left(f^{*}, f\right)_{W} \Rightarrow \\
\int_{\mathbb{R}}\left\langle f^{*}, f\right\rangle \delta \lambda d x=\int_{\mathbb{R}}\left\langle(l[u] f)^{\prime *} \cdot f^{*}, \delta u\right\rangle d x,
\end{gathered}
$$

whence

$$
\operatorname{grad} \lambda_{k}\left[f, u, f^{*}\right]=\left(0, f_{k}^{*} f_{k}, 0\right)^{\top}
$$

under normalization conditions

$$
\xi_{k}:=\int_{\mathbb{R}^{R}} d x\left\langle f_{k}^{*}, f_{k}\right\rangle=1
$$

for all $k=\overline{0, N(\lambda)}$. The analogous estamations for the augmented functionals $\gamma_{j} \in \mathcal{D}(W \times M \times W), j \in \overline{0, N(\gamma)}$, give the following obvious result:

$$
\operatorname{grad} \gamma_{j}\left[f, u, f^{*}\right]=\left(0, \operatorname{grad} \gamma_{j}[u], 0\right)^{\tau}
$$

for all $u \in M, f, f^{*} \in W$. Due to the linear nature of the equations (11.2) we can claim [35] that the vector field (11.1),(11.2) on the extended manifold $W \times M \times W$ is also Hamiltonian if the initially given vector field (11.1) is that on the manifold $M$. But due to the Lax representation (11.4) of the dynamical system(11.1) on $M$, this dynamical system is also Hamiltonian with respect to the Lie-Poisson bracket on the adjoint linear space $\hat{\mathcal{G}}^{*}$ of the Lie algebra $\hat{\mathcal{G}}$ of pseudodifferential operators on the complex Sobolev space $W$. Thereby we have [35] that the dynamical system (11.1), (11.2) on the manifold $W \times M \times W$ can be transformed into the Hamiltonian one with respect to the following implectic structure:

$$
\theta\left[f, l, f^{*}\right]:=\left(\begin{array}{ccc}
0 & 0 & -1 \\
0 & \theta[u] & 0 \\
1 & 0 & 0
\end{array}\right) .
$$

Therefore, we can try to reduce the implectic structure (11.10) on the nonlocal invariant submanifold $M_{N}^{\prime} \subset W \times M \times W$ with the set of constraints (11.8). To do this we have to take care for the set of functionals (11.8) to be included additionally into (11.5) as Lagrangian constraints. Hereof we get the following expressions determining the local manifold $M_{N} \subset W \times M \times W$

$$
M_{N}:=\left\{\left(f, u, f^{*}\right) \in M_{N}^{\prime}: \int_{\mathbb{R}^{*}} d x\left\langle f_{k}^{*}, f_{k}\right\rangle=1, k=\overline{1, N(\lambda)}\right\}
$$

being completely equivalent to the following:

$$
M_{N}=\left\{\left(f, u, f^{*}\right) \in W \times M \times W: \operatorname{grad} \mathcal{L}_{N}\left[f, u, f^{*}\right]=0\right\}
$$

where by the definition, $\mathcal{L}_{N}=\mathcal{L}_{N}^{\prime}+\sum_{k=0}^{N(\lambda)} c_{k}\left(\xi_{k}-1\right), c_{k} \in \mathbb{C}, K=\overline{\mathbf{0}, N(\lambda)}$, some arbitrary Lagrangian multipliers being determined on $M_{N}$ by the conditions $(11.8)$ in a unique way. Thereby we have got the finite dimensional 
local submanifold $M_{N} \subset W \times M \times W$ upon which the canonical symplectic structure $\Omega^{(2)} \in \Lambda^{2}\left(M_{N}\right)$ can be built successfully via the result of chapter (4) of this paper: if we have found the representation

$$
d \mathcal{L}_{N}\left[f, u, f^{*}\right]=\left\langle\operatorname{grad} \mathcal{L}_{N}\left[f, u, f^{*}\right],\left(d f, d u, d f^{*}\right)^{\tau}\right\rangle+d \alpha^{(1)}\left[f, u, f^{*}\right] / d x,
$$

where, by definition, $\mathcal{L}_{N}:=\int_{\mathbb{R}} d x \mathcal{L}_{N}\left[f, u, f^{*}\right] \in \mathcal{D}(W \times M \times W)$, we can construct on $M_{N}$ the canonical symplectic structure above mentioned as $\Omega^{(2)}=d \alpha^{(1)}$.

Let us proceed further to the reduction process of the dynamical system (11.1), (11.2) on the invariant already local submanifold $M_{N}(11.12)$. To do this let us recall that due to the construction above the Lagrangian $\mathcal{L}_{N}$ is a conservation law of the dynamical system (11.1),(11.2). Therefore, due to relations $d \mathcal{L}_{N} / d x=0, d \mathcal{L} / d t=0, x, t \in \mathbb{R}$, we obtain that

$$
\begin{gathered}
\left\langle\operatorname{grad} \mathcal{L}_{N}\left[f, u, f^{*}\right],\left(d f / d x, d u / d x, d f^{*} / d x\right)^{\tau}\right\rangle= \\
=-d h^{(x)}\left[f, u, f^{*}\right] / d x \\
\left\langle\operatorname{grad} \mathcal{L}_{N}\left[f, u, f^{*}\right],\left(p(l) f, K[u],-p^{*}(l) f^{*}\right)^{\tau}\right\rangle= \\
=-d h^{(t)}\left[f, u, f^{*}\right] / d x,
\end{gathered}
$$

where the scalar functions $h^{(x)}$ and $h^{(t)}$ on the manifold $M_{N}$ are the Hamiltonian ones for the vector fields $d / d x$ and $d / d t$ on $M_{N}$ respectively:

$$
d h^{(x)}=-i_{\frac{d}{d x}} \Omega^{(2)}, d h^{(t)}=-i_{\frac{d}{d t}} \Omega^{(2)}
$$

In the case of other vector fields $d / d \tau_{j}, j \in \overline{0, N(\gamma)}$, and $d / d t_{k}, k \in \overline{0, N(\lambda)}$, we can analogously obtain that

$$
\begin{gathered}
\left\langle\operatorname{grad} \mathcal{L}_{N}\left[f, u, f^{*}\right],\left(d f / d \tau_{j}, d u / d \tau_{j}, d f^{*} / d \tau_{j}\right)^{\tau}\right\rangle= \\
=-d h^{\left(\tau_{j}\right)}\left[f, u, f^{*}\right] / d x, \\
\left\langle\operatorname{grad} \mathcal{L}_{N}\left[f, u, f^{*}\right],\left(d f / d t_{k}, d u / d t_{k}, d f^{*} / d t_{k}\right)^{\tau}\right\rangle= \\
=-d h^{\left(t_{k}\right)}\left[f, u, f^{*}\right] / d x,
\end{gathered}
$$

where $d h^{\left(\tau_{j}\right)}=-i_{\frac{d}{d \tau_{j}}} \Omega^{(2)}, d h^{\left(t_{k}\right)}=-i_{\frac{d}{d t_{k}}} \Omega^{(2)}$ on $M_{N}$ for all $j \in \overline{0, N(\gamma)}, k \in$ $\overline{0, N(\lambda)}$. When obtaining the relationships (11.14)-(11.16), we have used as a matter of fact the spectral defining equations (11.3) for the left sides of (11.14)-(11.16) to be effectively represented as the full derivatives of some functionals. The latter note is the even needed to have been taken into consideration additionally to the regular procedure worked out in this article before. It is also easy to prove that all vector fields above $d / d \tau_{j}, j \in$ $Z_{+}, d / d t_{k}, k \in Z_{+}$, are commuting with each other: $\left\{\gamma_{j}, \lambda_{k}\right\}=\left\{\lambda_{k}, \lambda_{s}\right\}=$ $\left\{\gamma_{j}, \gamma_{l}\right\}=0$ for all $j, l \in \mathbb{Z}_{+}, k, s \in \overline{0, N(\lambda)}$.

\section{Example.}

Let us consider the dynamical system (11.1) on the functional manifold $M$, generated by the Lie-Poisson bracket on the coadjoint space of the Liealgebra $\mathcal{G}$ of pseudodifferential scalar operators on the real axis $\mathbb{R}$, and a Hamilton function $\gamma \in \mathcal{D}\left(\mathcal{G}^{*}\right)$ given for a simplicity as follows:

$$
\gamma=\bar{\gamma}=\frac{1}{k+1} \operatorname{Tr} l^{k+1}
$$



where $k \in Q$ - some rational number, $l \in \mathcal{G}^{*}$ - the Lax operator representable
as

$$
l=\sum_{j \gg-\infty)}^{u(l)} u_{j}(x) \xi^{j}, \quad \xi \simeq \partial / \partial x
$$

and satisfying the following general equation:

$$
d l / d t=\left[\operatorname{grad} \gamma(l)_{+}, l\right]-[\operatorname{grad} \gamma(l), l]_{+},
$$

where $\delta \gamma(l):=(\operatorname{grad} \gamma(l), \delta l)_{\mathcal{G}} . \quad(a, b)_{\mathcal{G}}:=\operatorname{tr}(a \circ b)$ for all $a, b \in \mathcal{G}$ by definition. If the functional $\gamma \in \mathcal{D}\left(\mathcal{G}^{*}\right)$ is due to (11.17) a Casimir one on the adjoint space $\mathcal{G}^{*}$, we obtain from (11.19) that

$$
d l . / d t=\left[\operatorname{grad} \gamma(l)_{+}, l\right] .
$$
If we define functions $f$ and $f^{*} \in W(\mathbb{R} ; \mathbb{C})$ as the eigenfunctions to the Lax
spectral equations

$$
\text { lf }=\lambda f . \quad l^{*} f^{*}=\lambda f^{*}, \quad \lambda \in \mathbb{C},
$$

the corresponding to $(11.20)$ evolution equations hold:

$$
d f / d t=l_{+}^{k} f . \quad d f^{*} / d t=-\left(l_{+}^{k}\right)^{*} f^{*} .
$$

The system of equations (11.20) and (11.22) on the extended phase space $\mathcal{G}^{*} \oplus W^{2}(\mathbb{R} ; \mathbb{C})$ constitutes the dynamical system which we try to represent as a Hamiltonian system. To do this, we have to use the Hamiltonian (11.17) and to demand it to depend on the function phase space $W^{2}$ explicitely, that is. $l=l\left[u ; f . f^{*}\right] \in \mathcal{G}^{*}$. Further. for the dynamical system (11.22) to be the Hamiltonian one in reference to the canonical symplectic structure on $W^{2}$, we need the following relationships to be satisfied:

$$
\delta \gamma(l) / \delta f=\left(l_{+}^{k}\right)^{*} f^{*} . \quad \delta \gamma(l) / \delta f^{*}=l_{+}^{k} f,
$$
for all $f, f^{*} \in W$. From $(11.17)$ and $(11.23)$ we therewith gain correspond-
ingly that

$$
\begin{gathered}
\delta \gamma(l):=\left(l^{k}, \delta l\right)_{\mathcal{G}}=\left(\left(l_{+}^{k}\right)^{*} f^{*}, \delta f\right)_{W}+\left(l_{+}^{k} f, \delta f^{*}\right)_{W}= \\
\left(f^{*}, l^{k} \delta f \xi^{-1}\right)_{\mathcal{G}}+\left(l^{k} \delta \xi^{-1}, \delta f^{*}\right)_{\mathcal{G}}= \\
\left(l^{k}, \delta f \xi^{-1} f^{*}+f \xi^{-1} \delta f^{*}\right)_{\mathcal{G}}=\left(l^{k}, \delta\left(f \xi^{-1} f^{*}\right)\right)_{\mathcal{G}} .
\end{gathered}
$$

Having equated the left and right hands of equations (11.24), we can state that the reduced on $W^{2}$ variation $\delta l \equiv \delta\left(f \xi^{-1} f^{*}\right)$, or $l\left[u ; f, f^{*}\right]=l[u]+$
f

$$
l=\sum_{j \gg-\infty}^{n(l ;} u_{j}(x) \xi^{j}+f \xi^{-1} f^{*}
$$

for all $f, f^{*} \in W$. Therefore, the following theorem is proved.

Theorem 5. Let some element $l \in \mathcal{G}^{*}$ be representable as the one in the form (11.25). Then the corresponding dynamical system (11.20) and $(11.22)$ on the phase space $\mathcal{G}^{*} \oplus W^{2}$ is the Hamiltonian system with relation 
to the canonical Lie-Poisson bracket on $\mathcal{G}^{*}$ and symplectic structure on $W^{2}$, correspondingly.

Our further step is to find the corresponding symplectic structure on the phase space $\mathcal{G}^{*} \oplus W^{2}$ for the case when the element $l \in \mathcal{G}^{*}$ is given by the expression (11.18). This problem is solved in a very simple way as it has been done in [35]: we need just to make the canonical Poisson structure

$$
\left(\begin{array}{c}
\delta \gamma / \delta l \\
\delta \gamma / \delta f \\
\delta \gamma / \delta f^{*}
\end{array}\right) \rightarrow\left(\begin{array}{c}
{\left[\delta \gamma / \delta l_{+}, l\right]-\left[\delta \gamma / \delta l, l_{+}\right.} \\
\delta \gamma / \delta f^{*} \\
-\delta \gamma / \delta f
\end{array}\right)
$$

on the phase space $\mathcal{G}^{*} \oplus W^{2}$ into a. Poisson structure on itself subject to the change of coordinates on $\mathcal{G}^{*} \oplus W^{2}$ :

$$
\left(l ; f, f^{*}\right)^{\tau} \rightarrow\left(l-f \xi^{-1} f^{*} ; f, f^{*}\right)
$$

As a result of the transformation (11.27) above we can easily find that the corresponding Poisson structure on the fase space $\mathcal{G}^{*} \oplus W^{2}$ gets the following form [35]:

$$
\left(\begin{array}{c}
\delta \gamma / \delta l \\
\delta \gamma / \delta f \\
\delta \gamma / \delta f^{*}
\end{array}\right) \rightarrow\left(\begin{array}{c}
{\left[\delta \gamma / \delta l_{+}, l\right]-[\delta \gamma / \delta l, l]_{+}-f \xi^{-1}(\delta \gamma / \delta f)+\left(\delta \gamma / \delta f^{*}\right) \xi^{-1} f^{*}} \\
(\delta \gamma / \delta l \cdot f)_{+}+\delta \gamma / \delta f^{*} \\
-\left((\delta \gamma / \delta l)^{*} f^{*}\right)_{+}-\delta \gamma / \delta f
\end{array}\right)
$$

Hence, the following theorem is true.

Theorem 6. Dynamical system $(11.19),(11.22)$ on the phase space $\mathcal{G}^{*} \oplus W^{2}$ is the Hamiltonian one with respect to the Poisson structure (11.28), the Lax spectral element $l \in \mathcal{G}^{*}$ being chosen in the form (11.18).

The further Theorem gives rise, for example, to a solution of the following important problem: what is the evolution of the eigenfunctions for the spectral value problem (11.21) when the Hamiltonian functional is given as follows:

$$
\gamma(l)=\bar{\gamma}+\bar{\lambda}
$$

where $\bar{\lambda} \in \mathcal{D}\left(\mathcal{G}^{*} \times W^{2}\right)$ - an eigenvalue of the spectral value problem (11.21). Indeed, using the simply derived formula

$$
\operatorname{grad} \bar{\lambda}=\left(\bar{f} \xi^{-1} \bar{f}^{*} ; l^{*} j^{*}, l \bar{f}\right)^{\tau}
$$

where $f, \bar{f}^{*} \in W$ - the corresponding eigenfunctions, the element $l \in \mathcal{G}^{*}$ is given by $(11.18)$, one obtains, due to the Poisson bracket $(11.28)$ on $\mathcal{G}^{*} \oplus W^{2}$, the following result:

$$
\begin{aligned}
d l / d t & =\left[l_{+}^{k}, l\right]-\left[\bar{f} \xi^{-1} \bar{f}^{*}, l\right]_{+}, \\
d \bar{f} / d t & =l_{+}^{k} \bar{f}+l \bar{f} \\
d \bar{f}^{*} / d t & =-\left(l_{+}^{k}\right)^{*} \bar{f}^{*}-l^{*} \bar{f}^{*}
\end{aligned}
$$

The hierarchy of dynamical systems $(11.31)$ on the phase spase $\mathcal{G}^{*} \oplus W^{2}$ for all $k \in Z_{+}$when reduced upon an invariant submanifold $M_{N} \subset M$ generates the commuting finite dimensional Liouville integrable vector fields on $M_{N} \subset M \times W^{2}$ subject to the canonical symplectic structure regularly 
built before. It is obvious also that the hierarchy (11.31) is easily generalized to the case when the Hamiltonian (11.29) takes the following form:

$$
\gamma(l)=\sum_{k=0}^{N(\gamma)} \frac{c_{k}}{k+1} t r l^{k+1}+\sum_{q=0}^{N(\lambda)} s_{q} \tilde{\lambda}_{q},
$$

where $c_{k} \in \mathbb{R}, k=\overline{0, N(\gamma)}$, and $s_{q} \in \mathbb{R} \cdot q=\overline{0, N(\lambda)}$ - some arbitrary but fixed real numbers. As a result from (11.28) we find that

$$
\begin{aligned}
& d l / d t=\sum_{k=0}^{N(\gamma)} c_{k}\left[l_{+}^{k}, l\right]-\sum_{q=0}^{N(\lambda)} s_{q}\left[\bar{f}_{q} \xi^{-1} \bar{f}_{q}^{*}, l\right] \\
& d \bar{f}_{j} / d t=\sum_{k=0}^{N(\gamma)} c_{k} l_{+}^{k} \bar{f}_{j}+s_{j} l \bar{f}_{j}, \\
& d \bar{f}_{j}^{*} / d l=-\sum_{k=0}^{N(\gamma)} c_{k}\left(l_{+}^{k}\right)^{*} \bar{f}_{j}^{*}-s_{j} l^{*} \bar{f}_{j}^{*}
\end{aligned}
$$

where $l \bar{f}_{j}=\bar{\lambda}_{j} f_{j}, l^{*} f_{j}=\bar{\lambda}_{j} f_{j}^{*}$ for all $j=\overline{1, N(\lambda)}$. The dynamical systems (11.34) are also commuting with each other Liouville integrable Hamiltonial flows on each Euler-Lagrange type invariant submanifold $M_{N} \subset M \times W^{2 N(\lambda)}$ with respect to the canonical symplectic structure on $M_{N} \subset M \times W^{2 N(\lambda)}$. Here we need just to mention that the whole theory of Neumann type integrable dynamical systems on submanifolds like the spheres $S^{N}$ and others are contained in the theory having been developed in the present article. Some applications being interested in plasma physics, nonlinear optics and others fields of science we are going to display in great detail in subsequent paper by authors.

\section{Conclusion}

The developed above theory of parametrically Lax-type integrable dynamical systems concedes to widen to a great extent the class of exactly treated nonlinear models in many fields of science. It is to be noted here the following important mathematical fact got in the paper: almost every nonlinear dynamical system admits a parametrically isospectral Lax type representation but a given dynamical system is the Lax-type integrable if an evolution of the spectrum parameter doesn't depend on a point $u \in M$ at all Cauchy data. This result has allowed us to develop a very effective direct criterium for the following problem to be solved: whether a given nonlinear dynamical system on the functional manifold $M$ is parametrically Lax-type integrable or not. Having the problem above solved, we have suggested the reduction. procedure for the associated nonlinear dynamical systems to be descended. on the invariant submanifold $M_{N} \subset M$ built before inheriting the canonical Hamiltonian structure and the Liouville complete integrability. Thereby, the powerful techniques of perturbation theory can be successfully used for dynarnical systems under consideration, as well as the relationships between the full Hamiltonian theory and various Hamiltonian truncations could be now got understandable more deeply. 
The imbedding problem for infinite-dimencional dynamical systems with additional structures such as invariants and symmetries is as old as the Newton-Lagrange mechanics, having been treated by many researches, using both analytical and algebraic methods. The powerfull differential-geometric tools used here were created mainly in works by E. Cartan at the beginning of the twentieth century. The great impact in the development of imbedding methods was done in last lime, especially owing the inventure the theory of isospectral deformations for some linear structures, built on the special vector bundles over the spase $M$ as the base of a given nonlinear dynamical system. Among them there are such structures as the moment map $l: M \rightarrow$ $\mathcal{G}^{*}$ into the adjoint space to the Lie algebra $\mathcal{G}$ of symmetries, acting on the symplectic phase space $M$ equivariantly $[8.10]$, the connection of the CartanEresman structures appearing via the Wahlquist-Estabrook approach [11], and many others.

For the last years the general structure of Lagrangian and Hamiltonian formalisms was studied thoroughly using both geometrical and algebraical methods $[12,13]$. The special attention was paid to the theory of differentialdifference dynamical systems on the infunite-dimensional manifolds [13.14]. Some number of articles was devoted to the theory of pure discrete dynamical systems [15-18] as well as treating the interesting examples [18] appeared to be important for applications.

In future work we intend to treat further imbedding problems for infinitedimensional both continuous and discrete dynamical systems basing on the differential-geometric Cartan's theory of differential ideals in Grassmann algebras over jet-manifolds, intimately connected with the problem under regard.

\section{References}

[1] P. A. Griffiths. Exterior Differential Systems and the Calculus of Variations. New York. Birkhause, 1982.480p.

[2] Prykarpatsky A. K., B. M. Fil. Category of topological jet-manifolds and certain applications in the theory of nonlinear infinite-dimensional dynamical systerns. // Ukr. Math. J., 1993, vol. 44, No 2 p. 1136-1147.

[3] P. D. Lax. Periodic solutions of the Korteweg-de Vries equation. // Comm. Pure and Appl. Mathem., 1968, vol. 21, No 2, p. 467-490.

[4] R. L. Bryant. On notions of Equivalence of Variational Problems with One Independent Variable. // Contemp. Math., 1987, vol. 63, p. 65-76.

[5] S. Stermberg. Some preliminary remarks on the formal variational calculus of Gel'fand and Dikii.// Lect. Notes in Mathem., 1980. vol. 150, p. 399-407.

[6] S. Steruberg. Lectures on differential geontetry. Moscow. Mir, 1961, p. 412.

[7] O. Bogoyavlensky, S. Novikov. On connection of Hamiltonian formalisms of stationar and nonstationar problenss. // Function. Anal., 1976, vol. 10, No 1, p. 9-13.

[8] Prykarpatsky A. K.. I. V. Mykytiuk. Algebraic aspects of integrable dynamical systems on manifolds. Kiev. Nruk. dumka, 1991, 380p.

[9] I. M. Gelfand, L. A. Dikiy Integrable nonlinear equations and Liouville theorem. // Function. Anal. Appl., 1979, vol. 13, No 1, p. 8-20.

[10] V.Gillemin, S.Stemberg. The moment map and Collective motion. // Ann. Phys., 1980 , vol. 127 , No 2 , p. $22(0-253$

[11] Wahlgnist H. D., Estabrook F. B. Prolongation structures of nonlinear evolution equations. // J. Math. Phys., 1975, vol. 16, No 1, p. 1-7, 1976, vol. 17, No 7, p. 1293-1297.

[12] Kupershmidt B. A. Geometry of jet-hundles and the structure of Lagrangian and Hammmiltonian formalisms. // Lecl. Notes Math., 1980, vol. 775, p. $162-218$ 
[13] Kupershmidt B. A. Discrete Lax equations and differential-difference calculus. // Asterisque, 1985, vol. 123, p. 5-212.

[14] Duft P. Li L.-C., Tomei C. Loop groups, discrete versions of some classical integrable systems and rank-2 extensions. // Memoirs of the AMS, 1992, vol. 100 , No 479 , p. $1-101$

[15] Moser J., Veselov A. P. Discrete versions of some classical integrable systems and factorization of matrix polynomials. Zurich, ETH, Preprint, 1989, 76p.

[16] Baez J. C., Gillam J. W. An algebraic approach to discrete mechanics. // Lett. Math. Phys., 1994, vol. 31, No 3, p. 205-212.

[17] Veselov A. P. What is an itegrable mapping? in: What is itegrability? Ney York, Springer-Verlag, 1991, p. 251-272.

[18] Levi D., Winternitz P. Continuous symmetries of discrete equations. // Physics Lett. A., 1991, vol. 152, No 7, p. 335-338.

[19] Kaneko K. Symplectic cellular automate. // Phys. Lett. A., 1988, vol. 129, No 1 , p. 9-16.

[20] Albraham R., Marsden J. Foundation of mechanics. London, The Blujamin Publish. Co., 1978, p. 806.

[21] Souriau J, M. Structure des systemes dynamique. Paris, Dunod, 1970.

[22] Adler M. On a trace functional for formal pseudo-differential operators and the symplectic structures of the Corteweg-de Vries equations. // Invent. Mathem., 1979 , vol. 50, No 2, p. 219-248.

[23] Prykarpatsky A.K. and others. Algebraic structure of the gradient-golonomic algorithm for Lax integrable nonlinear dynamical systems. // J. Math. Phys., vol. 35, No 4, p. 1763-1777, vol. 35, No 8, p. 6115-6126.

[24] Oewel W. Dirac constraints in field theory: Lifts of Hamiltonian systems to the cotangent bundle. // J. Math. Phys., 1988, vol. 29, No 1, p. 210-219.

[25] Oewel W. R-structures, Yang-Baxter equations and related involution theorerrs. // J. Math. Phys., 1989, vol. 30, No 5, p. 1140-1149.

[26] Fokas A. S., Gelfand 1. M. Bi-Hamiltonian Structures and Integrability. in: Important developments in Soliton Theory. Springer-Verlag, 1992.

[27] Olver P. J. Canonical forms and integrability of bi-Hamiltonian systems. // Phys. Lett. A., 1990, vol. 148, No 3, p. 177-187

[28] Magri F. A simple model of the integrable Hamiltonian equation. // J. Math. Phys., 1978, vol. 19, No 3, p. 1156-1162.

[29] Fernandes R. L. Completely integrable bi-Hamiltonian systems. // J. of Dynarr. and Diff. Equ., 1994, vol. 6, No 1, p. 53-69.

[30] Mitropolsky Yu. A., Bogoliubov N. N., Prykarpatsky A. K., Samoilenko V.H. Integrable dynamical systems. Spectral and differential-geometric aspects. Kiev, Naukova Dumka, 1987.

[31] S.P.Novikov (editor). The theory of solitons. Moscov, Mir, 1980.

[32] Mitropolsky Yu. O., Prykarpatsky A. K., Fil B. M. Some aspects of a gradientholonomic algorithm in the theory of integrability of nonlinear dynamical systems and computer algebra problems // Ukrainian Math. J., 1991, vol. 43, No 1, p. $63-74$.

[33] Kuybida V. V., Prytula M. M., Prykarpatsky A. K. The study of properties of the parametric isospectral integrability of nonlinear dynamical systems on functional manifolds and their finitedimensional approximations. Preprint of Inst. for Appl. Problems of Mech. and Mathem. of Ukr. Academy of Sci., Lviv, 1991, No 10, 41p.

[34] Marsden J., Weinstein A. Reduction of symplectic manifolds with symmetries. // Rep. Math. Phys., 1974, vol. 5, No 2, p. 121-130.

[35] Oewel W., Strampp W. Conscrained KP-hierarchy and bi-Harniltonian structures. // Comm. Math. Phys., 1993, vol. 157, No 1, p. 51-81. 


\title{
ДЕЯКІ ЗАУВАЖЕННЯ ДО ЛАГРАНЖЕВОГО ТА ГАМІЛЬТОНОВОГО ФОРМАЛІЗМІВ У БЕЗМЕЖНОВИМІРНИХ ДИНАМІЧНИХ СИСТЕМАХ 3 CИMЕTPIEF
}

\author{
А.Прикарпатський, Р.Самуляк, Д.Блекмор, \\ В.Стрампп, Ю.Сидоренко
}

Запропоновано підхід до лагранжевого та гамільтонового формалізмів, що природнім чином використовують інваріантні структури нелінійних динамічних систем на нескінченновимірних функщіональних многовидах. Основні ідеї запозичені 3 теорії Картана диференціальних систем на асоціативних джетмноговидах. Структури симетрій редукованих на інваріантні підмноговиди критичних точок делкого нелокального функціоналу Ейлера-Лагранжа описані як у випадку циференціальних, так і у випадку дискретних пиференціально-різницевих динамічних систем. 